\title{
WEAK LIMITS OF STOPPED DIFFUSIONS ${ }^{1}$
}

BY

\author{
J. R. BAXTER, R. V. CHACON AND N. C. JAIN
}

\begin{abstract}
We consider the following homogenization problem: Let Brownian motion in $\mathbf{R}^{d}, d \geqslant 3$, be killed on the surface of many small absorbing bodies (standard diffusion equation with Dirichlet boundary conditions). We investigate the limit as the number of bodies approaches infinity and the size of the bodies approaches 0 . By taking a weak limit of stopping times we replace a convergence problem on the state space by an identification of the limit on the sample space. This technique then gives results without smoothness assumptions which were previously necessary.
\end{abstract}

1. Introduction. We wish to consider Brownian motion in dimension $d \geqslant 3$, with many small absorbing bodies $D_{1}, \ldots, D_{k}$ scattered in $\mathbf{R}^{d}$. The Brownian motion is to be stopped, or "killed", whenever it reaches one of these bodies. Following [6 and 7], we study the approximate density of the diffusion process in the complement of the absorbing bodies, in the limit, as the number of bodies becomes infinite and the size of the bodies goes to zero. In particular, we wish to present a new method for studying questions of this sort which leads to more general results.

In Papanicolaou and Varadhan [6], $d=3$ and a certain standard reference body $D$, which is compact, contains the origin in its interior, and has a $C^{1}$ boundary, is specified. The Newtonian capacity of $D$ is denoted by $\alpha$. For $n=1,2, \ldots$, points $x_{1}(n), \ldots, x_{n}(n)$ are given, and a small copy $D_{i}(n)$ of $D$,

$$
D_{i}(n)=\left\{y \in \mathbf{R}^{d}: n\left(y-x_{i}(n)\right) \in D\right\},
$$

is placed at $x_{i}(n), 1 \leqslant i \leqslant n$. Let $D_{n}=\bigcup_{i=1}^{n} D_{i}(n)$, and

$$
\tau_{n}=\inf \left\{t>0: B_{t} \in D_{n}\right\},
$$

where $B_{t}, t \geqslant 0$, denotes standard Brownian motion in $\mathbf{R}^{d}$, so $\tau_{n}$ is the first hitting time of $D_{n}$ by Brownian motion. Let $\mu_{i}(n)=(\alpha / n) \delta_{x_{i}(n)}$, where $\delta_{x}$ denotes the Dirac measure which gives mass 1 to the singleton $x$, and let $\mu_{n}=\sum_{i=1}^{n} \mu_{i}(n)$. The following assumptions are made in [6]:

$$
\mu_{n} \stackrel{w}{\rightarrow} \lambda \quad \text { as } n \rightarrow \infty,
$$

Received by the editors March 28, 1985.

1980 Mathematics Subject Classification. Primary 60G40, 60J45, 35K05.

Key words and phrases. Homogenization, stopping times, compactness.

${ }^{1}$ This work was partly supported by the National Science Foundation (U.S.A.) and the National Science and Engineering Research Council of Canada. 
where $\lambda$ is a measure with a continuous and compactly supported density $h$ in $\mathbf{R}^{d}$, and the convergence is the weak convergence of measures, i.e. $\int f d \mu_{n} \rightarrow \int f d \lambda$ for all bounded continuous $f: \mathbf{R}^{d} \rightarrow \mathbf{R}^{1}$. Also,

$$
\sum_{i \neq j}\left\langle\mu_{i}(n), \mu_{j}(n)\right\rangle \rightarrow\langle\lambda, \lambda\rangle \text { as } n \rightarrow \infty,
$$

where for any measure $\nu,\langle\nu, \nu\rangle$ denotes the electrostatic self-energy

$$
\langle\nu, \nu\rangle=\iint|x-y|^{2-d} d \nu(x) d \nu(y) .
$$

In particular, for $i \neq j$,

$$
\left\langle\mu_{i}(n), \mu_{j}(n)\right\rangle=\left(\alpha^{2} / n^{2}\right)\left|x_{i}(n)-x_{j}(n)\right|^{2-d} .
$$

If $f: \mathbf{R}^{d} \rightarrow \mathbf{R}^{1}$ is continuous with compact support, and

$$
\begin{aligned}
u_{n}(x, t) & =E^{x}\left[f\left(B_{t}\right) \chi_{\left[\tau_{n}>t\right]}\right], \\
u(x, t) & =E^{x}\left[f\left(B_{t}\right) \exp \left\{-\int_{0}^{t} h\left(B_{s}\right) d s\right\}\right],
\end{aligned}
$$

where $E^{x}$ denotes expectation with respect to Brownian motion started at $x$, then the main result in [6] is the following

Theorem 1.1 (Papanicolaou-Varadhan). If $d=3$ and (1.1) and (1.2) hold, then given $\varepsilon>0$ and $t_{0}>0$,

$$
\lim _{n \rightarrow \infty} m\left[x \in \mathbf{R}^{d}: \sup _{0 \leqslant t \leqslant t_{0}}\left|u_{n}(x, t)-u(x, t)\right|>\varepsilon\right]=0,
$$

where $m$ denotes the Lebesgue measure in $\mathbf{R}^{d}$.

REMARK 1.0. It should be pointed out that only vague convergence in (1.1) is assumed in [6]; but in that context, since the function $f$ is bounded and has compact support, without any loss of generality one may assume all sets $D_{n}$ to be contained in a bounded ball $B$, and then one can replace vague convergence by weak convergence.

We may regard $u_{n}(x, t)$ as the density at $(x, t)$ of the diffusion started with initial density $f$ if the diffusion is killed on $D_{n}$, and $u(x, t)$ as the density at $(x, t)$ of a diffusion with initial density $f$ if the diffusion is randomly killed at a rate $h(x)$ at $x$. If $\tau$ denotes a randomized stopping time corresponding to random killing at rate $h$, then we can also write

$$
u(x, t)=E^{x}\left[f\left(B_{t}\right) \chi_{[\tau>t]}\right] .
$$

Furthemore, if $\tau_{n}$ can be shown to converge to $\tau$ in an appropriate sense, then the above convergence of $u_{n}(x, t)$ to $u(x, t)$ would follow. Our approach is to make this idea precise. For this we need to prove some results on randomized stopping times, and we do this in $\$ 2$. For related ideas and results we refer to [1, 2, 4 and 5]. The main results of this paper are Theorems $1.2-1.5$ which will be stated below. Their proofs will be given in $\S 3$. Theorem 1.5 can be compared directly with Theorem 1.1 . 
In Theorems 1.4 and 1.5 we do not need the bodies $D_{i}(n)$ to be of the same shape or size, nor do we require that they have smooth boundaries; we also do not require $h$, the density of $\lambda$, to be continuous or to have compact support. Indeed, $\lambda$ can even be infinite. Another advantage of our approach is that the distributions of $B_{\tau_{n}}$ restricted to $\left[\tau_{n} \leqslant t\right]$ converge weakly to the corresponding distribution of $B_{\tau}$ restricted to $[\tau \leqslant t]$. In other words, we can tell in the limit where the absorbed mass of the diffusion is actually being held.

To state our results, we start with closed sets $D_{n}, n \geqslant 1$, in $\mathbf{R}^{d}$. Condition (1.1) (resp., (1.2)) will be replaced either by the "local" condition (1.3) (resp., (1.4)) or by the "global" condition (1.5) (resp., (1.6)). We now state these conditions. If $\nu$ is a measure in $\mathbf{R}^{d}$ and $B$ is a Borel set, we define the measure $\chi_{B} \nu$ by

$$
\chi_{B} \nu(A)=\nu(B \cap A),
$$

for any Borel set $A$; Pot $\nu$ denotes the Newtonian potential of $\nu$, i.e.

$$
\text { Pot } \nu(x)=\int|x-y|^{2-d} d \nu(y) .
$$

In the conditions below, $\lambda$ is a fixed measure in $\mathbf{R}^{d}$ with bounded density $h$.

(1.3) Given any bounded ball $B \subset \mathbf{R}^{d}$, there exists a sequence of finite measures $\left\{\mu_{n}^{B}\right\}$ such that $\mu_{n}^{B} \stackrel{w}{\rightarrow} \chi_{B} \lambda$, and there exists a sequence $0 \leqslant \varepsilon_{n}^{B} \rightarrow 0$ such that Pot $\mu_{n}^{B}(x) \geqslant 1+\operatorname{Pot} \chi_{B} \lambda(x)-\varepsilon_{n}^{B}$ for q.e. $x \in D_{n} \cap \bar{B}, n \geqslant 1$.

(1.4) Given any bounded ball $B \subset \mathbf{R}^{d}$, there exists a sequence of finite measures $\left\{\nu_{n}^{B}\right\}$, with suppoprt of $\nu_{n}^{B} \subset D_{n} \cap \bar{B}$, such that $\nu_{n}^{B} \stackrel{\text { w }}{\rightarrow} \chi_{B} \lambda$, and there exists a sequence $0 \leqslant \varepsilon_{n}^{B} \rightarrow 0$ such that $\operatorname{Pot} \nu_{n}^{B}(x) \leqslant 1+\operatorname{Pot} \chi_{B} \lambda(x)+\varepsilon_{n}^{B}$ for $x \in D_{n} \cap \bar{B}$, $n \geqslant 1$.

For the next two (global) conditions we require $\lambda$ to be finite, i.e. $\int h d m<\infty$.

(1.5) There exists a sequence of finite measures $\left\{\mu_{n}\right\}$ in $\mathbf{R}^{d}$ such that $\mu_{n} \stackrel{n^{\prime \prime}}{\rightarrow} \lambda$, and there exists a sequence $0 \leqslant \varepsilon_{n} \rightarrow 0$ such that $\operatorname{Pot} \mu_{n}(x)>1+\operatorname{Pot} \lambda(x)-\varepsilon_{n}$, for q.e. $x \in D_{n}, n \geqslant 1$.

(1.6) There exists a sequence of finite measures $\left\{\nu_{n}\right\}$ in $\mathbf{R}^{d}$, with support of $\nu_{n} \subset D_{n}$, such that $\nu_{n} \stackrel{w}{\rightarrow} \lambda$, and there exists a sequence $0 \leqslant \varepsilon_{n} \rightarrow 0$ such that Pot $\nu_{n}(x)<1+\operatorname{Pot} \lambda(x)+\varepsilon_{n}$, for $x \in D_{n}, n \geqslant 1$.

REMARK 1.1. Note that the inequalities in (1.4) and (1.6) actually hold for all $x$ by the domination principle.

REMARK 1.2. If $\lambda$ is finite and (1.5) and (1.6) hold, then by Lemma 3.4 the local conditions (1.3) and (1.4) hold.

Conversely, if $\lambda$ is finite and (1.4) holds, it follows easily from Remark 1.1 that (1.6) holds.

As before, $\tau_{n}=\inf \left\{t>0: B_{t} \in D_{n}\right\}$. For $x \in \mathbf{R}^{d}, t \geqslant 0, n \geqslant 1$, let the measures $\nu_{n, t}^{x}$ and $\nu_{t}^{x}$ be defined by

$$
\nu_{n, t}^{x}(A)=E^{x}\left[\chi_{A}\left(B_{t}\right) \chi_{\left[\tau_{n}>t\right]}\right]
$$


and

$$
\nu_{t}^{x}(A)=E^{x}\left[\chi_{A}\left(B_{t}\right) \exp \left\{-\int_{0}^{t} h\left(B_{s}\right) d s\right\}\right] .
$$

We will denote by $\|\nu\|$ the total variation norm of the measure $\nu$.

THEOREM 1.2. Let $\left\{D_{n}\right\}$ be a sequence of closed sets in $\mathbf{R}^{d}$ and let $\lambda$ be a (finite or infinite) measure with a bounded density $h$. If (1.3) and (1.4) hold, then for any bounded ball $B$, given $\varepsilon>0, t_{0}>0$

$$
\lim _{n \rightarrow \infty} m\left\{x \in B: \sup _{t \leqslant t_{0}}\left\|\nu_{n, t}^{x}-v_{t}^{x}\right\|>\varepsilon\right\}=0 .
$$

In the next theorem $\lambda$ is finite, and a global condition gives a global conclusion.

THEOREM 1.3. Let $\left\{D_{n}\right\}$ be a sequence of closed sets in $\mathbf{R}^{d}$, and let $\lambda$ be a finite measure with a bounded density $h$. If (1.4) and (1.5) hold, then for any $\varepsilon>0, t_{0}>0$

$$
\lim _{n \rightarrow \infty} m\left\{x \in \mathbf{R}^{d}: \sup _{t \leqslant t_{0}}\left\|\nu_{n, t}^{x}-v_{t}^{x}\right\|>\varepsilon\right\}=0 .
$$

Remark 1.3. By Lemma 3.4 we can replace (1.4) by (1.6) in Theorem 1.3.

Conditions such as (1.3) and (1.4) are hard to understand intuitively; they do not have the simple geometric appeal of conditions (1.1) and (1.2). We now specialize the above theorems in the context of (1.1) and (1.2). We assume that

$$
D_{n}=\bigcup_{i=1}^{k_{n}} D_{i}(n), \quad n \geqslant 1,
$$

where each $D_{i}(n)$ is compact, and $k_{n} \rightarrow \infty$. We assume that there exists a sequence $0<\rho_{n} \rightarrow 0$, a constant $C>0$, and a sequence of points $x_{i}(n), 1 \leqslant i \leqslant k_{n}$, such that

$$
\begin{aligned}
& \text { (i) } D_{i}(n) \subset B\left(x_{i}(n), \rho_{n}\right), \\
& \text { (ii) } C \alpha_{i}(n) \geqslant \rho_{n}^{d-2}, \quad 1 \leqslant i \leqslant k_{n}, n \geqslant 1,
\end{aligned}
$$

where $B(x, r)$ denotes the ball with center at $x$ and radius $r$, and $\alpha_{i}(n)=$ Newtonian capacity of $D_{i}(n)$. The conditions imposed on the bodies $D_{i}(n)$ in Theorem 1.1. clearly imply these conditions. Let

$$
\alpha_{n}=\sum_{i=1}^{k_{n}} \alpha_{i}(n), \quad \mu_{i}(n)=\alpha_{i}(n) \delta_{x_{i}(n)}, \quad \mu_{n}=\sum_{i=1}^{k_{n}} \mu_{i}(n) .
$$

Having stated the conditions on the structure of $D_{n}$, we now state the local and global analogues of conditions (1.1) and (1.2). We assume that $\lambda$ is a measure on $\mathbf{R}^{d}$ (finite or infinite) with a bounded density $h$. We do not assume $h$ to be continuous. The local conditions are the following:

(1.10) For each bounded ball $B, \chi_{B} \mu_{n} \stackrel{w}{\rightarrow} \chi_{B} \lambda$ as $n \rightarrow \infty$.

(1.11) For each bounded ball $B, \sum_{i \neq j}^{*}\left\langle\mu_{i}(n), \mu_{j}(n)\right\rangle \rightarrow\left\langle\chi_{B} \lambda, \chi_{B} \lambda\right\rangle$ as $n \rightarrow \infty$, where the asterisk on the sum indicates summation over those $i, j$ for which $x_{i}(n)$ and $x_{j}(n)$ are in $B$. 
If $\lambda\left(\mathbf{R}^{d}\right)<\infty$, then the global conditions are the following:

$$
\mu_{n} \stackrel{n}{\rightarrow} \lambda \text { as } n \rightarrow \infty
$$

and

$$
\sum_{i \neq j}\left\langle\mu_{i}(n), \mu_{j}(n)\right\rangle \rightarrow\langle\lambda, \lambda\rangle \quad \text { as } n \rightarrow \infty .
$$

THEOREM 1.4. If $\left\{D_{n}\right\}$, a sequence of compacts in $\mathbf{R}^{d}, d \geqslant 3$, satisfies (1.9), and (1.10) and (1.11) hold, then for any bounded ball $B$, any $\varepsilon>0$ and $t_{0}>0$, we have

$$
\lim _{n \rightarrow \infty} m\left\{x \in B: \sup _{t \leqslant t_{0}}\left\|\nu_{n, t}^{x}-\nu_{t}^{x}\right\|>\varepsilon\right\}=0
$$

where $\nu_{n, t}^{x}$ and $\nu_{t}^{x}$ are given by (1.7) and (1.8), respectively.

THEOREM 1.5. If $\left\{D_{n}\right\}$, a sequence of compacts in $\mathbf{R}^{d}, d \geqslant 3$, satisfies (1.9), and (1.12) and (1.13) hold, then for any $\varepsilon>0, t_{0}>0$, we have

$$
\lim _{n \rightarrow \infty} m\left\{x: \sup _{t \leqslant t_{0}}\left\|\nu_{n, t}^{x}-\nu_{t}^{x}\right\|>\varepsilon\right\}=0 .
$$

In the following sections $\|h\|$ will always denote the sup norm of the bounded function $h$.

2. Formulation in terms of randomized stopping times. Let $C$ denote the space of continuous functions from $[0, \infty)$ into $\mathbf{R}^{d}$ with the metric topology of uniform convergence on compacts. We will consider Brownian motion in $\mathbf{R}^{d}$ using $C$ as the sample space. Let the maps $B_{t}: C \rightarrow \mathbf{R}^{d}, t \geqslant 0$, be defined by $B_{t}(\omega)=\omega(t)$ for $\omega \in C$. Let $\mathscr{F}=\sigma\left\{B_{t}: t \geqslant 0\right\}, \mathscr{F}_{t}=\sigma\left\{B_{s}: s \leqslant t\right\}$ and let $\mathscr{G}_{t}=\mathscr{F}_{t+}=\bigcap_{s>t} \mathscr{F}_{s}$. We set $\mathscr{F}_{\infty}=\mathscr{F}=\mathscr{G}_{\infty}$. We will denote by $\mathscr{B}_{t}$ the Borel subsets of $[0, t]$ and by $\mathscr{B}$ the Borel subsets of $[0, \infty]$.

A stopping time $\tau$ with respect to the filtration $\left(\mathscr{G}_{t}\right)$ is a map $\tau: C \rightarrow[0, \infty]$ such that $[\tau \leqslant t] \in \mathscr{G}_{t}$ for all $t$. We will suppress mentioning the filtration unless it is different from $\left(\mathscr{G}_{t}\right)$. A randomized stopping time $\tau$ is a map $\tau: C \times[0,1] \rightarrow[0, \infty]$ such that $[\tau \leqslant t] \in \mathscr{G}_{t} \times \mathscr{B}_{1}$ for all $t$. We will adopt the convention that a randomized stopping time $\tau$ is nondecreasing and left-continuous in the second variable for all $\omega$. A stopping time $\tau$ can always be regarded as a randomized stopping time by extending $\tau$ from $C$ to $C \times[0,1]$ in the obvious way.

To each randomized stopping time $\tau$ one can associate a function $A: C \times \mathscr{B} \rightarrow$ $[0,1]$ in the following manner:

$$
\begin{aligned}
A(\omega,[0, t]) & =\sup \{v \leqslant 1: \tau(\omega, v) \leqslant t\} \\
& =0 \text { if the set is empty. }
\end{aligned}
$$

Since for a fixed $\omega$ this is nondecreasing in $t$ and equals 1 when $t=\infty$, we get a probability measure on $[0, \infty]$ and $A$ is defined on $C \times \mathscr{B}$. Clearly

$$
\tau(\omega, v)=\inf \{t \leqslant \infty: A(\omega,[0, t]) \geqslant v\}
$$


for $0 \leqslant v \leqslant 1, \omega \in C$. The function $A$ defined in (2.1) satisfies

$$
A(\omega, \cdot) \text { is a probability measure on }[0, \infty] \text { for } \omega \in C,
$$

and

$$
A(\cdot,[0, t]) \in \mathscr{G}_{t} \text { for all } t
$$

If $A$ is a function from $C \times \mathscr{B}$ to $[0,1]$ satisfying (2.3) and (2.4) and $\tau$ is defined by (2.2), then $\tau$ is a randomized stopping time. For this reason such a map $A$ is called a stopping time measure. If $\tau$ is a stopping time and $\hat{\tau}$ the associated randomized stopping time (extension of $\tau$ to $C \times[0,1]$ ) then $A$ corresponding to $\hat{\tau}$ is $A(\omega, \cdot)=$ $\delta_{\tau(\omega)}$. It will be convenient for us to work with $A$ rather than the randomized stopping time $\tau$. A more general discussion of stopping time measures is given in [1, 2].

In the rest of the paper all stopping times and stopping time measures will be with respect to the filtration $\left(\mathscr{G}_{t}\right)$.

Definition 2.1. If $P$ is a probability measure on $(C, \mathscr{F})$ and $A: C \times \mathscr{B} \rightarrow[0,1]$ is a stopping time measure, then for $D \in \mathscr{F} \times \mathscr{B}$ we define

$$
P \otimes A(D)=\int_{C} \int_{[0, \infty]} \chi_{D}(\omega, v) A(\omega, d v) P(d \omega)
$$

We call $P \otimes A$ the stopped process.

If $\tau$ is the randomized stopping time obtained from $A$ by (2.2), $I: C \times[0,1] \rightarrow C$ is the projection map, and $m$ denotes Lebesgue measure on $[0,1]$, then $P \otimes A$ is just the distribution of the map $(I, \tau): C \times[0,1] \rightarrow C \times[0, \infty]$ with respect to $P \times m$.

We note that for any $t$, any $Y \in L^{1}(C, \mathscr{F}, P)$, and any bounded $\mathscr{B}$-measurable $f$ : $[0, \infty) \rightarrow \mathbf{R}$ such that $f=0$ on $(t, \infty)$, we have

$$
\int Y f d Q=\int E\left(Y \mid \mathscr{G}_{t}\right) f d Q
$$

where $Q=P \otimes A$ and, as usual, a function defined on $C$ or $[0, \infty)$ is extended to $C \times[0, \infty)$ in the obvious way, and conditional expection is taken with respect to $P$, the marginal of $P \otimes A$ on $C$.

The following lemma gives a useful characterization of a stopped process; it is a variant of Lemma $2.9[\mathbf{1}]$.

LEMMA 2.1. Let $Q$ be a probability measure on $\mathscr{F} \times \mathscr{B}$ such that for each $t \geqslant 0$ the relation (2.6) holds for every $f:[0, \infty) \rightarrow \mathbf{R}$ continuous with support in $[0, t]$ and $Y$ : $C \rightarrow \mathbf{R}$ of the form $Y(\omega)=g_{1}\left(\omega\left(t_{1}\right)\right) \cdots g_{k}\left(\omega\left(t_{k}\right)\right)$, where $g_{i}: C_{0}\left(\mathbf{R}^{d}\right) \rightarrow \mathbf{R}, 0 \leqslant t_{i}<$ $\infty, 1 \leqslant i \leqslant k, k \geqslant 1$. Then there exists a unique stopping time measure $A$ such that $Q$ is the stopped process $P \otimes A, P$ being the marginal of $Q$ on $C$.

Proof. For $0 \leqslant t<\infty$, let

$$
c(t)=E^{Q}\left\{\chi_{[0, t]} \mid \mathscr{G}_{t} \times\{\varnothing,[0,1]\}\right\}, \quad c(\infty)=1,
$$


where the conditional expectation is with respect to $Q$. For a fixed $t$ we have $c(t)$ : $C \times[0,1] \rightarrow[0,1]$ and this map is $\mathscr{G}_{t} \times\{\varnothing,[0,1]\}$-measurable. Therefore $c(t)$ is a function of $\omega$ only and is $\mathscr{G}_{t}$-measurable. It follows that

$$
\int Y \chi_{[0, t]} d Q=\int Y c(t) d P, \quad Y \in L^{1}\left(C, \mathscr{G}_{t}, P\right) .
$$

For $s<t$ we have $c(s) \leqslant c(t)$ a.e. $(P)$. We first define $c$ to be nondecreasing on rationals for each $\omega$ and then define $c_{1}(t)=\inf \{c(s): s \geqslant t, s$ rational $\}$ for any $t$ and $\omega$, then $c_{1}(t)$ is right-continuous and nondecreasing in $t$ for each $\omega$ and (2.7) is satisfied with $c=c_{1}$ for all $t$ and all $Y \in L^{1}\left(C, \mathscr{G}_{t}, P\right)$. By the monotone class argument and the right-continuity of $\mathscr{G}_{t}$ our assumptions imply that (2.6) holds for all $Y \in L^{1}(C, \mathscr{F}, P)$ and all bounded measurable $f:[0, \infty) \rightarrow \mathbf{R}$ which vanish on $(t, \infty)$. Therefore for $Y \in L^{1}(C, \mathscr{F}, P)$,

$$
\int Y \chi_{[0, t]} d Q=\int E\left(Y \mid \mathscr{G}_{t}\right) \chi_{[0, t]} d Q
$$

and now by (2.7) this last equals $\int E\left(Y \mid \mathscr{G}_{t}\right) c_{1}(t) d P=\int Y c_{1}(t) d P$ since $c_{1}(t) \in \mathscr{G}_{t}$. It follows that (2.7) with $c=c_{1}$ holds for all $Y \in L^{1}(C, \mathscr{F}, P)$. We set

$$
A(\omega,[0, t])=c_{1}(t)(\omega) \text {. }
$$

Then $A$ is a stopping time measure and for $Y \in L^{1}(C, \mathscr{F}, P)$

$$
\begin{aligned}
\int Y \chi_{[0, t]} d Q & =\int Y A(\omega,[0, t]) d P \\
& =\int Y(\omega) \chi_{[0, t]}(s) d A(\omega, s) d P(\omega) \\
& =\int Y \chi_{[0, t]} d(P \otimes A) .
\end{aligned}
$$

The monotone class argument shows $Q=P \otimes A$, so the lemma is proved.

From now on $P^{x}$ will denote the probability measure on $(C, \mathscr{F})$ representing Brownian motion started at $x$, and $P^{\mu}$ will represent Brownian motion with initial distribution $\mu$. In this context these will be called Brownian probabilities.

LEMMA 2.2. Let $\left\{\mu_{n}\right\}$ be a sequence of probability measures on Borel subsets of $\mathbf{R}^{d}$. Let $P_{n}=P^{\mu_{n}}$. Suppose that $\left\{A_{n}\right\}$ is a sequence of stopping time measures such that $P_{n} \otimes A_{n}$ converges weakly on $C \times[0, \infty]$ to a limit $Q$. Then $\mu_{n} \stackrel{w}{\rightarrow} \mu, P_{n} \stackrel{w}{\rightarrow} P^{\mu}$ and $Q$ is a stopped process whose marginal on $C$ is $P^{\mu}$.

Proof. If $\left\{P_{n} \otimes A_{n}\right\}$ converges weakly on $C \times[0, \infty]$, then clearly $P_{n}$ converges weakly on $C$ to some probability measure $\hat{P}$. Since $P_{n}=P^{\mu_{n}}$, it is then clear that $\mu_{n} \stackrel{w}{\rightarrow}$ some $\mu$ and $\hat{P}=P^{\mu}$, which is the marginal of $Q$ on $C$. To check that $Q$ is a stopped process we will use Lemma 2.1 , so let $f:[0, \infty) \rightarrow \mathbf{R}$ be continuous with support in $[0, t]$ and $Y(\omega)=g_{1}\left(\omega\left(t_{1}\right)\right) \cdots g_{k}\left(\omega\left(t_{k}\right)\right)$, where $g_{i} \in C_{0}\left(\mathbf{R}^{d}\right)$. Let $t_{1}, \ldots, t_{r}$ be $\leqslant t$ and $t_{r+1}, \ldots, t_{k}$ be $>t$, and let $Z(\omega)=g_{1}\left(\omega\left(t_{1}\right)\right) \cdots g_{r}\left(\omega\left(t_{r}\right)\right)$, $W(\omega)=g_{r+1}\left(\omega\left(t_{r+1}\right)\right) \cdots g_{k}\left(\omega\left(t_{k}\right)\right)$. Then, since $P_{n} \otimes A_{n}$ is a stopped process, we 
have

$$
\int Y f d\left(P_{n} \otimes A_{n}\right)=\int E^{P_{n}}\left(Y \mid \mathscr{G}_{t}\right) f d\left(P_{n} \otimes A_{n}\right) .
$$

By the Markov property of Brownian motion

$$
E^{P_{n}}\left(Y \mid \mathscr{G}_{t}\right)=Z \varphi\left(B_{t}\right)
$$

where $\varphi(x)=E^{x}\left\{g_{r+1}\left(B_{t_{r+1}-t}\right) \cdots g_{k}\left(B_{t_{h}-t}\right)\right\}$. The maps $Y f$ and $Z \varphi\left(B_{t}\right) f$ are clearly continuous and bounded (as maps from $C \times[0, \infty)$ to R). Therefore, using (2.9) in (2.8) and letting $n \rightarrow \infty$ we get

$$
\int Y f d Q=\int Z \varphi\left(B_{t}\right) f d Q
$$

but we also have $Z \varphi\left(B_{t}\right)=E^{P^{\mu}}\left(Y \mid \mathscr{G}_{t}\right)$, so (2.10) implies that $Q$ is a stopped process by Lemma 2.1. This completes the proof.

We now introduce a condition such that if a sequence of stopped processes satisfying this condition converges weakly (to a stopped process, by Lemma 2.2) then the stopping time measure of the limit process has a definite representation. This is the content of Theorem 2.1 .

Condition A. Let $h: \mathbf{R}^{d} \rightarrow[0, \infty)$ be a bounded Borel measurable function and $I \subset[0, \infty)$ be an interval. A family $\left\{P^{\mu} \otimes A_{n}: \mu \in \Gamma_{n}, n \geqslant 1\right\}$ of stopped processes will be said to satisfy Condition A on the interval $I$ with respect to $h$, if there exists a function $g:[0, \infty) \rightarrow[0, \infty)$ which satisfies $\lim _{t \downarrow 0}(g(t) / t)=0$, and for $t \geqslant 0$, $\Delta>0$, such that $[t, t+\Delta] \subset I$, there exists a sequence $\varphi_{n}^{t, \Delta}: \mathbf{R}^{d} \rightarrow[0, \infty)$ of Borel measurable functions which satisfies

$$
\sup _{\substack{n \geq 1 \\ x \in \mathbf{R}^{d}}} \varphi_{n}^{t, \Delta}(x)<\infty ; \quad \lim _{n \rightarrow \infty} m\left\{B \cap\left\{x: \varphi_{n}^{t, \Delta}(x)>\eta\right\}\right\}=0
$$

for every bounded ball $B$ and $\eta>0$, and for $\mu \in \Gamma_{n}, n \geqslant 1$, we have

$$
\left|E^{\mu}\left\{A_{n}((t, t+\Delta]) \mid \mathscr{G}_{t}\right\}-A_{n}((t, \infty]) h_{\Delta}\left(B_{t}\right)\right| \leqslant g(\Delta)+\varphi_{n}^{t, \Delta}\left(B_{t}\right),
$$

$P^{\mu}$-a.s., where we write

$$
h_{\Delta}(x)=E^{x} \int_{0}^{\Delta} h\left(B_{s}\right) d s .
$$

REMARK 2.1. (i) The inequality in (2.12) asserts that the quantities

$$
E^{\mu}\left\{A_{n}((t, t+\Delta]) \mid \mathscr{G}_{t}\right\} \text { and } A_{n}((t, \infty]) h_{\Delta}\left(B_{t}\right)
$$

are approximately equal, with an error made up of two parts: $g(\Delta)$, which becomes very small as $\Delta \rightarrow 0$, and $\varphi_{n}^{t, \Delta}\left(B_{t}\right)$ which becomes small for "most" paths as $n \rightarrow \infty$.

(ii) The sets $\Gamma_{n}$ of probability measures are introduced in Condition A to keep it sufficiently flexible for the theorems that we are going to prove in this section. In Theorem 2.1 we have $\Gamma_{n}$ consisting of the singleton $\mu_{n}$, whereas in Theorem 2.3 we take $\Gamma_{n}=\left\{\delta_{x}: x \in \mathbf{R}^{d}\right\}, n \geqslant 1$.

(iii) If the $\tau_{n}$ 's are hitting times of Brownian motion, then as remarked earlier $A_{n}((t, t+\Delta])=\chi_{\left[t<\tau_{n} \leqslant t+\Delta\right]}$. Suppose for each $\Delta>0$, there exists a sequence $\varphi_{n}^{\Delta}$ : $\mathbf{R}^{d} \rightarrow[0, \infty)$ which satisfies (2.11) and for all $x \in \mathbf{R}^{d}, n \geqslant 1$, we have

$$
\left|P^{x}\left\{0 \leqslant \tau_{n} \leqslant \Delta\right\}-h_{\Delta}(x)\right| \leqslant g(\Delta)+\varphi_{n}^{\Delta}(x),
$$


where $g:[0, \infty) \rightarrow[0, \infty)$ satisfies $\lim _{t \downarrow 0}(g(t) / t)=0$; then Condition A is satisfied for $\Gamma_{n}=$ set of all probability measures on $\mathbf{R}^{d}$ with $\varphi_{n}^{t, \Delta}=\varphi_{n}^{\Delta}$. This follows because if $\mu$ is any probability measure on $\mathbf{R}^{d}$, then by the Markov property of Brownian motion, writing $p_{n}^{\Delta}=P^{x}\left[0 \leqslant \tau_{n} \leqslant \Delta\right]$, we have

$$
E^{\mu}\left\{A_{n}((t, t+\Delta]) \mid \mathscr{G}_{t}\right\}=\chi_{\left[\tau_{n}>t\right]} p_{n}^{\Delta}\left(B_{t}\right) .
$$

Thus (2.12) becomes

$$
\chi_{\left[\tau_{n}>t\right]}\left|p_{n}^{\Delta}\left(B_{t}\right)-h_{\Delta}\left(B_{t}\right)\right| \leqslant g(\Delta)+\varphi_{n}^{t, \Delta}\left(B_{t}\right),
$$

which clearly holds if (2.14) is satisfied. In our present application we will be able to check the stronger condition (2.14).

THEOREM 2.1. Let $\left\{\mu_{n}\right\}$ be a sequence of probability measures in $\mathbf{R}^{d}$ and $P_{n}=P^{\mu_{n}}$ be Brownian probabilities on $(C, \mathscr{F})$. Let $\left\{A_{n}\right\}$ be a sequence of stopping time measures such that the sequence $\left\{Q_{n}\right\}=\left\{P_{n} \otimes A_{n}\right\}$ satisfies Condition A (with $\left.\Gamma_{n}=\left\{\mu_{n}\right\}\right)$ with respect to $h$ on the interval $I=(0, T)$, where $0<T \leqslant \infty$. Suppose that $\left\{Q_{n}\right\}$ converges weakly on $C \times[0, \infty]$ to $Q=P \otimes$ A. Then a.s. $(P)$

$$
A((t, \infty])=A((0, \infty]) \exp \left(-\int_{0}^{t} h\left(B_{s}\right) d s\right), \quad t \in[0, T) .
$$

For the proof of this theorem it will be convenient to separate a part of the argument into the following

LEMMA 2.3. Under the conditions of Theorem 2.1, if $0<t<\infty$ and $0<\Delta<\infty$ are such that $(t, t+\Delta] \subset I$, then for any $\mathscr{G}_{t}$-measurable $Y: C \rightarrow \mathbf{R},|Y| \leqslant 1$, we have

$$
\left|\int Y A((t, t+\Delta]) d P-\int Y A((t, \infty]) h_{\Delta}\left(B_{t}\right) d P\right| \leqslant g(\Delta)
$$

furthermore, if $0 \leqslant t \leqslant u<T$, we have

$$
\int Y A((t, u]) d P=\iint_{t}^{u} Y A((s, \infty]) h\left(B_{s}\right) d s d P,
$$

where $h_{\Delta}$ is defined in (2.13).

Proof. Let $t, \Delta$ be given as above and fix $\delta>0$ such that $t+\Delta+\delta / 2<T$. Let $\psi$ and $\rho$ be continuous functions on $[0, \infty]$ such that $\psi=0$ on $[0, t+\delta / 2] \cup[t+\Delta$ $+\delta / 2, \infty], \psi=1$ on $[t+\delta, t+\Delta], \psi$ linear elsewhere; $\rho=0$ on $[0, t], \rho=1$ on $[t+\delta / 2, \infty]$ and $\rho$ linear elsewhere. By the monotone class argument it is enough to consider $0 \leqslant Y \leqslant 1, Y$ continuous on $C$, to prove (2.15). Then

$$
\begin{aligned}
\int Y \psi d Q_{n} & =\int Y \int \psi(s) d A_{n}(s) d P_{n} \\
& \leqslant \int Y A_{n}\left(\left(t+\frac{\delta}{2}, t+\Delta+\frac{\delta}{2}\right]\right) d P_{n} \\
& \leqslant \int Y A_{n}\left(\left(t+\frac{\delta}{2}, \infty\right]\right) h_{\Delta}\left(B_{t+\delta / 2}\right) d P_{n}+g(\Delta)+\int \hat{\varphi}_{n}\left(B_{t+\delta / 2}\right) d P_{n},
\end{aligned}
$$


where (2.12) is used for the second inequality and $\hat{\varphi}_{n}=\varphi_{n}^{t+\delta / 2 .\lrcorner . ~ T h e r e f o r e ~ w e ~ g e t ~}$

$$
\begin{aligned}
\int Y \psi d Q_{n} & \leqslant \int Y h_{\Delta}\left(B_{t+\delta / 2}\right) \int \rho(s) d A_{n}(s) d P_{n}+g(\Delta)+\int \hat{\varphi}_{n}\left(B_{t+\delta / 2}\right) d P_{n} \\
& =\int Y h_{\Delta}\left(B_{t+\delta / 2}\right) \rho d Q_{n}+g(\Delta)+\int \hat{\varphi}_{n}\left(B_{t+\delta / 2}\right) d P_{n} .
\end{aligned}
$$

It is easy to see that $h_{\Delta}: \mathbf{R}^{d} \rightarrow \mathbf{R}$ is bounded and continuous, so $h_{\Delta}\left(B_{t+\delta / 2}\right): C \rightarrow \mathbf{R}$ is bounded continuous. Also, since $Q_{n} \stackrel{n}{\rightarrow} Q$, by Lemma 2.2 we have $\mu_{n} \stackrel{n}{\rightarrow} \mu$, therefore (2.11) implies that $\int \hat{\varphi}_{n}\left(B_{t+\delta / 2}\right) d P_{n} \rightarrow 0$ as $n \rightarrow \infty$. Therefore, letting $n \rightarrow \infty$ we get

$$
\int Y \psi d Q \leqslant \int Y h_{\Delta}\left(B_{t+\delta / 2}\right) \rho d Q+g(\Delta) .
$$

Now letting $\delta \rightarrow 0$ we get (dominated convergence)

$$
\int Y A((t, t+\Delta]) d P \leqslant \int Y A((t, \infty]) h_{\Delta}\left(B_{t}\right) d P+g(\Delta) .
$$

A similar argument shows the reverse inequality with $g(\Delta)$ replaced by $-g(\Delta)$ on the right side. 'This proves (2.15).

To prove (2.16), for any $k=1,2, \ldots$, let $k \Delta=u-t$ and $t_{j}=t+j \Delta, 0 \leqslant j \leqslant k$, so $t_{0}=t$ and $t_{k}=u$. Let $J_{1}$ denote the left side in (2.16) and $J_{2}$ the right side. Let

$$
\begin{aligned}
J_{3} & =\sum_{j=0}^{k-1} \int Y A\left(\left(t_{j}, \infty\right]\right) h_{\Delta}\left(B_{t}\right) d P \\
& =\sum_{j=0}^{k-1} \int Y A\left(\left(t_{j}, \infty\right]\right) \int_{t_{j}}^{t_{j+1}} h\left(B_{s}\right) d s d P .
\end{aligned}
$$

We can write

$$
J_{1}=\sum_{j=0}^{k-1} \int Y A\left(\left(t_{j}, t_{j+1}\right]\right) d P
$$

and then by (2.15) we have

$$
\left|J_{1}-J_{3}\right| \leqslant k g(\Delta) \text {. }
$$

Also,

$$
J_{2}=\sum_{j=0}^{k-1} \int Y \int_{t_{j}}^{t_{j+1}} A((s, \infty]) h\left(B_{s}\right) d s d P .
$$

Using the second expression for $J_{3}$ we get

$$
\left|J_{2}-J_{3}\right| \leqslant \sum_{j=0}^{k-1} \iint_{t_{j}}^{t_{j+1}} A\left(\left(t_{j}, s\right]\right) d s d P\|h\| .
$$

By (2.15) with $Y=1$ we get

$$
\int A((t, t+\Delta]) d P \leqslant\|h\| \Delta+g(\Delta) .
$$


Using this in (2.18) gives

$$
\left|J_{2}-J_{3}\right| \leqslant k \Delta^{2}\|h\|^{2}+k \Delta g(\Delta)\|h\| .
$$

Therefore (2.17) and (2.20) give

$$
\left|J_{1}-J_{2}\right| \leqslant k g(\Delta)+k \Delta^{2}\|h\|^{2}+k \Delta g(\Delta)\|h\| .
$$

As $k \rightarrow \infty$, the right side tends to 0 and (2.16) is proved.

Proof of Theorem 2.1. Let $A_{t}=A((t, \infty])$ and

$$
Z_{t}=A_{t} \exp \left(\int_{0}^{t} h\left(B_{s}\right) d s\right)
$$

The theorem will be proved by showing that $\left\{Z_{t}, 0 \leqslant t<T\right\}$ is a martingale with filtration $\left(\mathscr{G}_{t}\right)$. Let

$$
\Phi_{t, u}=\exp \left(\int_{t}^{u} h\left(B_{s}\right) d s\right), \quad t \leqslant u .
$$

To show that $\left(Z_{t}, \mathscr{G}_{t}\right)$ is a martingale, it will suffice to show that for any $v \in[0, T)$, and any $\mathscr{G}_{v}$-measurable $Y, 0 \leqslant Y \leqslant 1$, the function

$$
\zeta(t)=\int Y Z_{t} d P, \quad v \leqslant t<T,
$$

is constant. Let $v \leqslant t \leqslant u<T$. Then

$$
\begin{aligned}
\zeta(u)-\zeta(t)= & \int Y \Phi_{0, t} \Phi_{t, u} A_{u} d P-\int Y \Phi_{0, t} A_{u} d P \\
& +\int Y \Phi_{0, t} A_{u} d P-\int Y \Phi_{0, t} A_{t} d P \\
= & I_{1}-I_{2},
\end{aligned}
$$

where

$$
I_{1} \equiv \int Y \Phi_{0, t} A_{u}\left(\Phi_{t, u}-1\right) d P=\int Y \Phi_{0, t} A_{u}\left(\int_{t}^{u} h\left(B_{s}\right) d s+W\right) d P
$$

and

$$
|W|=\left|\Phi_{t, u}-1-\int_{t}^{u} h\left(B_{s}\right) d s\right| \leqslant \frac{1}{2}\left(\int_{t}^{u} h\left(B_{s}\right) d s\right)^{2} \Phi_{t, u}
$$

because $e^{x}-1-x \leqslant \frac{1}{2} x^{2} e^{x}$ for $x \geqslant 0$, so

$$
|W| \leqslant \frac{1}{2}(u-t)^{2}\|h\|^{2} \exp (\|h\|(u-t)) .
$$

Also, by (2.16), which clearly holds for bounded $\mathscr{G}_{i}$-measurable $Y$,

$$
I_{2} \equiv \int Y \Phi_{0, t}\left(A_{u}-A_{t}\right) d P=\int Y \Phi_{0, t} \int_{t}^{u} A_{s} h\left(B_{s}\right) d s d P .
$$


Thus, noting that $\Phi_{0, t} \leqslant \exp (t\|h\|)$, we have

$$
\begin{aligned}
|\zeta(u)-\zeta(t)| & =\left|I_{1}-I_{2}\right| \\
& \leqslant \int Y \Phi_{0, t} \int_{t}^{u}\left(A_{s}-A_{u}\right) h\left(B_{s}\right) d s d P+\frac{1}{2}(u-t)^{2}\|h\|^{2} \exp (\|h\| u) .
\end{aligned}
$$

By (2.16) with $Y=1$ we get

$$
\int A((t, s]) d P \leqslant\|h\|(s-t), \quad t \leqslant s<T,
$$

and using this in (2.21) we get

$$
|\zeta(u)-\zeta(t)| \leqslant \frac{1}{2} e^{t\|h\|}\|h\|^{2}(u-t)^{2}+\frac{1}{2} e^{u\|h\| \|}\|h\|^{2}(u-t)^{2} .
$$

Thus $\zeta$ is Hölder continuous on $[v, T)$ with exponent 2 , and hence must be constant, proving that $\left(Z_{t}\right)$ is a martingale with filtration $\left(\mathscr{G}_{t}\right)$ on $[0, T)$. Since $\left(Z_{t}\right)$ has right-continuous paths and $\left(\mathscr{G}_{t}\right)$ is a Brownian motion filtration, by Theorem 2.VII.4 of [3] the martingale $\left(Z_{t}\right)$ must have continuous paths a.s. Clearly $\left(Z_{t}\right)$ has paths of bounded variation, so a.s. we have $Z_{t}=Z_{0}$ for $0 \leqslant t<T$. This proves the theorem.

RemarK 2.2. It should be noted that if $\int A(\{0\}) d P=0$, then $h$ determines $A$ uniquely in Theorem 2.1 .

The following corollary of Theorem 2.1 will be useful for applications.

Corollary 2.1. Let $\left\{\mu_{n}\right\},\left\{P_{n}\right\}$ and $\left\{A_{n}\right\}$ be as in Theorem 2.1, and suppose Condition A (with $\Gamma_{n}=\left\{\mu_{n}\right\}$ ) is satisfied by $\left\{Q_{n}\right\}=\left\{P_{n} \otimes A_{n}\right\}$ with respect to $h$ on $(0, \infty)$. Under these conditions if $\mu_{n} \stackrel{n}{\rightarrow} \mu$ and

$$
\lim _{\delta \rightarrow 0}\left[\liminf _{n} \int A_{n}([0, \delta]) d P_{n}\right]=0
$$

then $Q_{n} \stackrel{w}{\rightarrow} Q=P \otimes A$, and

$$
A((t, \infty])=\exp \left(\int_{0}^{t} h\left(B_{s}\right) d s\right), \quad t \geqslant 0 .
$$

Proof. Since $\mu_{n} \stackrel{w}{\rightarrow} \mu$, we have $P_{n} \stackrel{w}{\rightarrow} P^{\mu}$. Thus $\left\{Q_{n}\right\}$ is a tight sequence on $C \times[0, \infty]$, so to prove the corollary it suffices to show that every weakly convergent subsequence has the limit law defined above. Thus it is sufficient to assume that $P_{n} \otimes A_{n} \stackrel{w}{\rightarrow} P^{\prime} \otimes A^{\prime}$ and then show that $P^{\prime} \otimes A^{\prime}=P^{\mu} \otimes A$. We have already noted that $P_{n} \stackrel{n^{\prime}}{\rightarrow} P^{\mu}$, so $P^{\prime}=P^{\mu}$. Now $P_{n} \otimes A_{n} \stackrel{w}{\rightarrow} P^{\mu} \otimes A^{\prime}$ implies that for each $\delta>0$

$$
\int A^{\prime}([0, \delta)) d P^{\mu} \leqslant \liminf _{n} \int A_{n}([0, \delta)) d P_{n},
$$

therefore by (2.22) we have $\int A^{\prime}(\{0\}) d P^{\mu}=0$ and by Remark 2.1 then $A^{\prime}=A$ a.s. $\left(P^{\mu}\right)$. This proves the corollary.

The next theorem shows that weak convergence of stopped processes on the sample space implies very strong convergence for distributions on the state space.

It should be noted that the measures $\nu_{n, t}$ and $\nu_{t}$ defined in (2.23) and (2.24) are exactly the measures $\nu_{n, t}^{x}, \nu_{t}^{x}$ defined in (1.7) and (1.8), if we take $P_{n}=P^{x}$ for all $n$, and let $A_{n}$ be the stopping time measure associated with the hitting time $\tau_{n}$ of $\S 1$. 
THEOREM 2.2. Let $P_{n}=P^{\mu_{n}}$ and let $\left\{A_{n}\right\}$ be a sequence of stopping time measures such that $P_{n} \otimes A_{n} \stackrel{w}{\rightarrow} P \otimes A$. Let $v_{n, t}$ and $\nu_{t}$ be the Borel measures on $\mathbf{R}^{d}$ such that for any bounded Borel function $H: \mathbf{R}^{d} \rightarrow \mathbf{R}$

$$
\int_{\mathbf{R}^{d}} H d \nu_{n, t}=\int_{C} H\left(B_{t}\right) A_{n}((t, \infty]) d P_{n},
$$

and

$$
\int_{\mathbf{R}^{d}} H d \nu_{t}=\int_{C} H\left(B_{t}\right) A((t, \infty]) d P .
$$

If $0 \leqslant t_{n} \rightarrow t_{0} \in[0, \infty)$, and

$$
\int A\left(\left\{t_{0}\right\}\right) d P=0
$$

then $\nu_{n, t_{n}} \rightarrow \nu_{t_{0}}$ in the total variation norm when $t_{0}>0$ and weakly when $t_{0}=0$.

Proof. We first consider $t_{0}=0$. Let $\varepsilon>0$. By (2.25) there exists $\Delta>0$ such that $\int A([0, \Delta]) d P<\varepsilon$. Then by the weak convergence of $P_{n} \otimes A_{n}$ to $P \otimes A$ we have $n_{0}$ such that for $n \geqslant n_{0}, \int A_{n}([0, \Delta]) d P_{n}<\varepsilon$. Let $\mu_{n, s}$ denote the distribution of $B_{s}$ under $P_{n}$ and $\mu_{s}$ the distribution of $B_{s}$ under $P$. Then we have $\left\|\nu_{s}-\mu_{s}\right\|<\varepsilon$ and $\left\|\nu_{n, s}-\mu_{n, s}\right\|<\varepsilon$ for all $s \in[0, \Delta]$ and all $n \geqslant n_{0}$; so $\nu_{n, t_{n}} \rightarrow \nu_{0}$ weakly since $\mu_{n, t_{n}} \rightarrow \nu_{0}$ weakly.

Now assume $t_{0}>0$. Let $\varepsilon>0$ be fixed. By (2.25) and weak convergence there exists $\Delta>0$ such that $3 \Delta<t_{0}$ and for $n \geqslant n_{0}$

$$
\int A\left(\left[t_{0}-3 \Delta, t_{0}+\Delta\right]\right) d P<\varepsilon, \quad \int A_{n}\left(\left[t_{0}-3 \Delta, t_{0}+\Delta\right]\right) d P_{n}<\varepsilon .
$$

Let $f$ be defined on $[0, \infty)$ such that $f=\chi_{\left(t_{0}-3 \Delta, t_{0}-2 \Delta\right)} / \Delta$, and let $H: \mathbf{R}^{d} \rightarrow \mathbf{R}$ be Borel, $|H| \leqslant 1$. Then

$$
\begin{aligned}
\mid \int H d \nu_{n, t_{n}}-\int H( & \left.B_{t_{n}}\right) \int_{0}^{\infty} f(s) A_{n}((s, \infty]) d s d P_{n} \mid \\
& =\left|\int H\left(B_{t_{n}}\right) \int_{0}^{\infty} f(s)\left[A_{n}((s, \infty])-A_{n}\left(\left(t_{n}, \infty\right]\right)\right] d s d P_{n}\right| \\
& \leqslant \int_{0}^{\infty} f(s) \int A_{n}\left(\left(s, t_{n}\right]\right) d P_{n} d s<\varepsilon
\end{aligned}
$$

for all $n$ such that $\left|t_{n}-t_{0}\right|<\Delta$ and for which (2.26) holds. Similarly

$$
\left|\int H d \nu_{t_{0}}-\int H\left(B_{t_{0}}\right) \int_{0}^{\infty} f(s) A((s, \infty]) d s d P\right|<\varepsilon .
$$

Thus it is enough to prove that

$$
\int H\left(B_{t_{n}}\right) \int_{0}^{\infty} f(s) A_{n}((s, \infty]) d s d P_{n} \rightarrow \int H\left(B_{t_{0}}\right) \int_{0}^{\infty} f(s) A((s, \infty]) d s d P,
$$


as $n \rightarrow \infty$ uniformly over all Borel $H$ with $|H| \leqslant 1$. Furthermore, since $\left\{P_{n}\right\}$ is tight, it is enough to show that the convergence is uniform over any collection $S$ of functions $H$ which vanish outside a fixed bounded ball and $|H| \leqslant 1$. Taking conditional expectation with respect to $\mathscr{F}_{t_{n}-\Delta}$ first, we get

$$
\int H\left(B_{t_{n}}\right) \int_{0}^{\infty} f(s) A_{n}((s, \infty]) d s d P_{n}=\int G\left(B_{t_{n}-\Delta}\right) \int_{0}^{\infty} f(s) A_{n}((s, \infty]) d s d P_{n}
$$

for all $n$ such that $t_{n}>t_{0}-\Delta$, where $G(x)=E^{x}\left(H\left(B_{\Delta}\right)\right)$. For a given collection $S$ of the sort of $H$ 's described above let $S^{\prime}$ denote the corresponding collection of $G$ 's. $S^{\prime}$ is compact in the sup-norm topology. Thus it is enough to prove convergence in (2.27) for one function $H$. Now

$$
\int G\left(B_{t_{n}-\Delta}\right) \int_{0}^{\infty} f(s) A_{n}((s, \infty]) d s d P_{n}=\int G\left(B_{t_{n}-\Delta}\right) \int F(s) A_{n}(d s) d P_{n}
$$

where $F(s)=\int_{s}^{\infty} f(u) d u$. For each $G$, as $n \rightarrow \infty$

$$
\begin{aligned}
\int G\left(B_{t_{n}-\Delta}\right) \int F(s) A_{n}(d s) d P_{n} & \rightarrow \int G\left(B_{t_{0}-\Delta}\right) \int F(s) A(d s) d P \\
& =\int G\left(B_{t_{0}-\Delta}\right) \int_{0}^{\infty} f(s) A((s, \infty]) d s d P \\
& =\int H\left(B_{t_{0}}\right) \int_{0}^{\infty} f(s) A((s, \infty]) d s d P .
\end{aligned}
$$

This proves the theorem.

This theorem has the following

CorollaRy 2.2. Suppose $P_{n}=P^{\mu}$ for $n \geqslant 1$ and $P^{\mu} \otimes A_{n} \stackrel{n^{*}}{\rightarrow} P^{\mu} \otimes A$. If

$$
\int A(\{t\}) d P^{\mu}=0, \quad 0 \leqslant t<\infty,
$$

then for any $0<t_{0}<\infty$ we have

$$
\lim _{n} \sup _{0 \leqslant t \leqslant t_{0}}\left\|\nu_{n, t}-\nu_{t}\right\|=0
$$

Proof. Let $t_{n} \rightarrow t_{0}$. If $t_{0}>0$, then Theorem 2.2 applied to the two sequences $\left\{P^{\mu} \otimes A_{n}\right\}$ and $\left\{P^{\mu} \otimes A\right\}$ shows that $\left\|\nu_{n, t}-\nu_{t_{n}}\right\| \rightarrow 0$ as $n \rightarrow \infty$, which proves (2.29). If $t_{0}=0$, then the proof of Theorem 2.2 shows that $\left\|\nu_{n, t_{n}}-\nu_{t_{n}}\right\| \rightarrow 0$ again, so the corollary follows.

We can combine the two corollaries to prove the following

THEOREM 2.3. Suppose $\left\{A_{n}\right\}$ is a sequence of stopping time measures and $\left\{P^{x} \otimes A_{n}\right.$ : $\left.x \in \mathbf{R}^{d}, n \geqslant 1\right\}$ satisfies Condition A with respect to $h$ on $[0, \infty)$ (with $\Gamma_{n}=\left\{\delta_{x}\right.$ : $\left.x \in \mathbf{R}^{d}\right\}$ for all $n$ ), and for every bounded ball $B$, all $\varepsilon>0, \eta>0$,

$$
\lim _{n} m\left\{B \cap\left[x: P^{x}\left(A_{n}(\{0\})>\varepsilon\right)>\eta\right]\right\}=0 .
$$


Let

$$
A((t, \infty])=\exp \left(-\int_{0}^{t} h\left(B_{s}\right) d s\right), \quad t \geqslant 0,
$$

and let $\nu_{n, t}^{x}, \nu_{t}^{x}$ be the measures on $\mathbf{R}^{d}$ defined in (2.23) and (2.24) with $P_{n}=P=P^{x}$. Then for any $\varepsilon>0, t_{0}>0$, and bounded ball $B$, we have

$$
\lim _{n} m\left\{x \in B: \sup _{0 \leqslant t \leqslant t_{0}}\left\|\nu_{n, t}^{x}-\nu_{t}^{x}\right\|>\varepsilon\right\}=0 .
$$

Proof. Let the bounded ball $B$ and $t_{0}>0$ be fixed. It suffices to check that every subsequence $\left\{n^{\prime}(j)\right\}$ has a further subsequence $\{n(i)\}$ such that for $m$-a.e. $x$ in $B$

$$
\lim _{i} \sup _{0 \leqslant t \leqslant t_{0}}\left\|\nu_{n(i), t}^{x}-\nu_{t}^{x}\right\|=0 .
$$

Let the subsequence $\left\{n^{\prime}(j)\right\}$ be given. For any $x$ and $\delta>0$, by Condition A we have

$$
\begin{aligned}
\int A_{n}([0, \delta]) d P^{x} & \leqslant \int A_{n}(\{0\}) d P^{x}+g(\delta)+\delta\|h\|+\int \varphi_{n}^{0, \delta}\left(B_{0}\right) d P^{x} \\
& =\int A_{n}(\{0\}) d P^{x}+g(\delta)+\delta\|h\|+\varphi_{n}^{0, \delta}(x) .
\end{aligned}
$$

Conditions (2.11) and (2.30) imply that there exists a subsequence $\{n(i)\}$ of $\left\{n^{\prime}(j)\right\}$ such that

$$
\int A_{n(i)}(\{0\}) d P^{x}+\varphi_{n(i)}^{0, \delta}(x) \rightarrow 0 \text { for } m-\text { a.e. } x \text { in } B .
$$

Therefore for $m$-a.e. $x$ in $B$ we have

$$
\underset{i}{\limsup } \int A_{n(i)}([0, \delta]) d P^{x} \leqslant g(\delta)+\delta\|h\| .
$$

Thus for $m$-a.e. $x$ in $B$ the condition (2.22) for Corollary 2.1 is satisfied, so the corollary implies that for $m$-a.e. $x$ in $B$ we have $P^{x} \otimes A_{n(i)} \stackrel{w}{\rightarrow} P^{x} \otimes A$ with $A$ given by (2.31). Since this $A$ satisfies (2.28), by Corollary 2.2 we conclude that for $m$-a.e. $x$ in $B$ we have $\lim _{n(i)} \sup _{0 \leqslant t \leqslant t_{0}}\left\|\nu_{n(i), t}^{x}-\nu_{t}^{x}\right\|=0$, and the theorem is proved.

\section{Proofs of theorems of $\$ 1$.}

Proof OF THEOREM 1.2. The proof consists of showing that if (1.3) and (1.4) hold, then with $A_{n}$ corresponding to $\tau_{n}=\inf \left\{t>0: B_{t} \in D_{n}\right\}$, the conditions of Theorem 2.3 are satisfied. Our first step is to check Condition $A$ in the context of Theorem 2.3. We need the following

Lemma 3.1. Let $K$ be a closed subset of $\mathbf{R}^{d}$, and let

$$
\tau(\omega)=\inf \left\{t>0: B_{t}(\omega) \in K\right\} .
$$

Let $\Delta>0$ be fixed, and for $A$ a Borel subset of $\mathbf{R}^{d}, x \in \mathbf{R}^{d}$, let

$$
\varphi_{x}(A)=P^{x}\left\{B_{\tau} \in A ; \tau \leqslant \Delta\right\}, \quad \psi_{x}(A)=P^{x}\left\{B_{\Delta} \in A ; \tau \leqslant \Delta\right\} .
$$


Then for any $f \in L^{1}\left(\mathbf{R}^{d}\right)$ and $x \in \mathbf{R}^{d}$ we have

$$
\begin{gathered}
\int\left(\operatorname{Pot} \varphi_{x}(y)-\operatorname{Pot} \psi_{x}(y)\right) f(y) d m(y)=E^{x} \int_{\tau \wedge \Delta}^{\Delta} f\left(B_{s}\right) d s, \\
\operatorname{Pot} \varphi_{x}(y) \geqslant \operatorname{Pot} \psi_{x}(y), \quad y \in \mathbf{R}^{d}, \\
\operatorname{Pot} \varphi_{x}(y)-\operatorname{Pot} \psi_{x}(y) \leqslant \int_{0}^{\Delta} p(s, x, y) d s, \quad y \in \mathbf{R}^{d},
\end{gathered}
$$

where $p(s, x, y)=(2 \pi s)^{-d / 2} \exp \left\{-|x-y|^{2} / 2 s\right\} ;$ furthermore, for any $x$ in $\mathbf{R}^{d}$ equality holds in (3.3) quasi-everywhere (q.e.) on $K$.

Proof. Let

$$
\mu_{x}(A)=P^{x}\left\{B_{\Delta} \in A ; \tau>\Delta\right\}
$$

Then

$$
\varphi_{x}(A)+\mu_{x}(A)=P^{x}\left\{B_{\tau \wedge \Delta} \in A\right\}, \quad \psi_{x}(A)+\mu_{x}(A)=P^{x}\left\{B_{\Delta} \in A\right\},
$$

consequently, if $f \in L^{1}\left(\mathbf{R}^{d}\right)$, we have

$$
\int \operatorname{Pot}\left(\varphi_{x}+\mu_{x}\right)(y) f(y) d m(y)=E^{x} \int_{\tau \wedge \Delta}^{\infty} f\left(B_{s}\right) d s
$$

and

$$
\int \operatorname{Pot}\left(\psi_{x}+\mu_{x}\right)(y) f(y) d m(y)=E^{x} \int_{\Delta}^{\infty} f\left(B_{s}\right) d s .
$$

The quantities on the right side in (3.5) and (3.6) are dominated by $E^{x} \int_{0}^{\infty}|f|\left(B_{s}\right) d s$ $<\infty$, therefore (3.1) follows by subtracting (3.6) from (3.5). Since for all nonnegative $f \in L^{1}\left(\mathbf{R}^{d}\right)$ the left side in (3.1) is nonnegative, we conclude that (3.2) hold a.e. $(m)$, and since

$$
\operatorname{Pot} \mu(y)=\liminf _{\substack{z \rightarrow y \\ z \neq y}} \operatorname{Pot} \mu(z)
$$

for any superharmonic potential we must have (3.2) holding everywhere. For $f \geqslant 0$, (3.1) also gives

$$
\begin{aligned}
\int\left(\operatorname{Pot} \varphi_{x}-\operatorname{Pot} \psi_{x}\right)(y) f(y) d m(y) & \leqslant E^{x} \int_{0}^{\Delta} f\left(B_{s}\right) d s \\
& =\iint_{0}^{\Delta} p(s, x, y) d s d m(y)
\end{aligned}
$$

so (3.3) holds a.e. $(m)$ and then (3.7) again allows us to conclude inequality in (3.3) everywhere. Since $\mu_{x}+\varphi_{x}$ is the swept measure of $\delta_{x}$ onto $K$,

$$
\operatorname{Pot}\left(\mu_{x}+\varphi_{x}\right)=\operatorname{Pot} \delta_{x} \quad \text { q.e. on } K \text {. }
$$

By (3.6) and (3.7) we have for all $y$

$$
\operatorname{Pot}\left(\mu_{x}+\psi_{x}\right)(y)=\int_{\Delta}^{\infty} p(s, x, y) d s,
$$

and (3.8) and (3.9) imply that for any $x \in \mathbf{R}^{d}$ equality in (3.3) holds q.e. on $K$. This proves the lemma. 
The next two lemmas will easily imply the conditions of Theorem 2.3, namely, Condition A for $\left\{P^{x} \otimes A_{n}: x \in \mathbf{R}^{d}, n \geqslant 1\right\}$ and (2.30).

LEMMA 3.2. Let $\tau_{n}=\inf \left\{t>0: B_{t} \in D_{n}\right\}$. If (1.3) holds, then there exists $g_{1}$ : $[0, \infty) \rightarrow[0, \infty)$ such that $\lim _{t \downarrow 0}\left(g_{1}(t) / t\right)=0$, and for every $\Delta>0$, there exists $a$ sequence of Borel measurable functions $\left\{\varphi_{1, n}^{\Delta}\right\}$ which satisfies (2.11), such that for $x \in \mathbf{R}^{d}$

$$
P^{x}\left\{\tau_{n} \leqslant \Delta\right\} \leqslant h_{\Delta}(x)+g_{1}(\Delta)+\varphi_{1, n}^{\Delta}(x),
$$

where $h_{\Delta}$ is defined in (2.13).

Proof. Let $B(x, \varepsilon)$ denote the ball with center $x$ and radius $\varepsilon$. Let $\left\{C_{i}\right\}$ be a locally finite measurable partition of $\mathbf{R}^{d}$ such that there exists a countable set $\left\{\chi_{i}\right\} \subset \mathbf{R}^{d}$ with the property that $C_{i} \subset B\left(x_{i}, \frac{1}{2}\right)$. For $\Delta \geqslant 0$, let

$$
g_{1}(\Delta)=P^{0}\left\{\sup _{0 \leqslant t \leqslant \Delta}\left|B_{t}\right| \geqslant \frac{1}{2}\right\} \text {. }
$$

Then $g_{1}(\Delta) / \Delta \rightarrow 0$ as $\Delta \rightarrow 0$. Let $B(i)=B\left(x_{i}, 1\right), D_{n}^{i}=D_{n} \cap \bar{B}(i), \tau_{n}^{i}=\inf \{t>0$ : $\left.B_{t} \in D_{n}^{i}\right\}$. Let $\mu_{n}^{i}=\mu_{n}^{B(i)}, \lambda^{i}=\chi_{B(i)} \lambda$, denote the measures which satisfy (1.3) for $B=B(i)$, and in the same context let $\varepsilon_{n}^{i}=\varepsilon_{n}^{B(i)}$. For $x \in C_{i}$ we define $\varphi_{x, n}^{i}, \psi_{x, n}^{i}$, and $\mu_{x, n}^{i}$ as in Lemma 3.1 with $K=D_{n} \cap \bar{B}(i)$.

If $x \in C_{i}$, then $x \in B\left(x_{i}, \frac{1}{2}\right)$, and it follows that $P^{x}$-a.s.

Thus we have for $x \in C_{i}$

$$
\left\{\tau_{n} \leqslant \Delta ; \tau_{n}^{i}>\Delta\right\} \subset\left\{\sup _{0 \leqslant t \leqslant \Delta}\left|B_{t}-B_{0}\right|>\frac{1}{2}\right\} .
$$

$$
P^{x}\left\{\tau_{n} \leqslant \Delta\right\} \leqslant P^{x}\left\{\tau_{n}^{i} \leqslant \Delta\right\}+g_{1}(\Delta) .
$$

Now by (1.3) we have

$$
\begin{aligned}
P^{x}\left\{\tau_{n}^{i} \leqslant \Delta\right\} & =\int_{D_{n}^{i}} d \varphi_{x, n}^{i} \leqslant \int\left(\operatorname{Pot} \mu_{n}^{i}-\operatorname{Pot} \lambda^{i}+\varepsilon_{n}^{i}\right) d \varphi_{x, n}^{i} \\
& \leqslant \varepsilon_{n}^{i}+\int \operatorname{Pot} \varphi_{x, n}^{i} d \mu_{n}^{i}-\int \operatorname{Pot} \varphi_{x, n}^{i} d \lambda^{i} \\
& \leqslant \varepsilon_{n}^{i}+\int\left(\operatorname{Pot} \varphi_{x, n}^{i}-\operatorname{Pot} \psi_{x, n}^{i}\right) d \mu_{n}^{i}+\int \operatorname{Pot} \psi_{x, n}^{i}\left(d \mu_{n}^{i}-d \lambda^{i}\right),
\end{aligned}
$$

where we used (3.2) at the last step. By (3.3) we get for $x \in C_{i}$

$$
\begin{aligned}
P^{x}\left\{\tau_{n}^{i} \leqslant \Delta\right\} \leqslant & \varepsilon_{n}^{i}+\iint_{0}^{\Delta} p(s, x, y) d s d \mu_{n}^{i}(y) \\
& +\int \operatorname{Pot} \psi_{x, n}^{i}\left(d \mu_{n}^{i}-d \lambda^{i}\right) . \\
= & \varepsilon_{n}^{i}+\iint_{0}^{\Delta} p(s, x, y) d s d \lambda^{i}(y) \\
& +\iint_{0}^{\Delta} p(s, x, y) d s\left(d \mu_{n}^{i}(y)-d \lambda^{i}(y)\right) \\
& +\int \operatorname{Pot} \psi_{x, n}^{i}\left(d \mu_{n}^{i}-d \lambda^{i}\right) .
\end{aligned}
$$


Therefore

$$
\begin{aligned}
P^{x}\left\{\tau_{n}^{i} \leqslant \Delta\right\} & \leqslant \iint_{0}^{\Delta} p(s, x, y) d s d \lambda(y)+\varphi_{n}^{\Delta}(x) \\
& =h_{\Delta}(x)+\varphi_{1, n}^{i, \Delta}(x),
\end{aligned}
$$

where for $x \in C_{i}$

$$
\begin{aligned}
\varphi_{1, n}^{i, \Delta}(x)= & \varepsilon_{n}^{i}+\iint_{0}^{\Delta} p(s, x, y) d s\left(d \mu_{n}^{i}(y)-d \lambda^{i}(y)\right) \\
& +\int \operatorname{Pot} \psi_{x, n}^{i}\left(d \mu_{n}^{i}-d \lambda^{i}\right)
\end{aligned}
$$

We define

$$
\varphi_{1, n}^{\Delta}(x)=\sum_{i}\left\{\left|\varphi_{1, n}^{i, \Delta}(x)\right| \wedge(1+\Delta\|h\|)\right\} \chi_{C_{1}}(x) .
$$

It is clear that (3.11) holds for $x \in C_{i}$ if $\varphi_{1, n}^{i, \Delta}(x)$ is replaced by $\varphi_{1, n}^{\Delta}(x)$. This together with (3.10) will prove the lemma if we show that $\varphi_{1, n}^{\Delta}(x)$ satisfies (2.11). For this it suffices to show that for each $i$, as $n \rightarrow \infty$

$$
\int_{C_{i}}\left|\varphi_{1, n}^{i, \Delta}(x)\right| d m(x) \rightarrow 0 \text {. }
$$

To examine the convergence of $\int \operatorname{Pot} \psi_{x, n}^{i}\left(d \mu_{n}^{i}-d \lambda^{i}\right)$, we note that $\psi_{x, n}^{i} \leqslant \gamma_{x, \Delta}$, where $\gamma_{x, \Delta}$ is the distribution of $B_{\Delta}$ with respect to $P^{x}$, i.e. $d \gamma_{x, \Delta}(y)=$ $P(\Delta, x, y) d m(y)$. Therefore there exists a constant $c(\Delta)$, depending only on $\Delta$, such that $\operatorname{Pot} \psi_{x, n}^{i}(y) \leqslant c(\Delta)$ for all $i, x, n, y$. Furthermore, since $\left|\nabla \operatorname{Pot} \psi_{x, n}^{i}\right|$ is also bounded, we see that $\left\{\operatorname{Pot} \psi_{x, n}^{i}\right\}$ is uniformly equicontinuous. Hence

$$
\int \operatorname{Pot} \psi_{x, n}^{i}(y)\left(d \mu_{n}^{i}(y)-d \lambda^{i}(y)\right) \rightarrow 0 \quad \text { as } n \rightarrow \infty
$$

boundedly in $x$. It follows that

$$
\lim _{n \rightarrow \infty} \int_{C_{i}}\left|\int \operatorname{Pot} \psi_{x, n}^{i}(y)\left(d \mu_{n}^{i}(y)-d \lambda^{i}(y)\right)\right| d m(x)=0 .
$$

Since $\varepsilon_{n}^{i} \rightarrow 0$ as $n \rightarrow \infty$, to check (3.13) it only remains to show that

$$
\lim _{n \rightarrow \infty} \int_{C_{i}}\left|\iint_{0}^{\Delta} p(s, x, y) d s\left(d \mu_{n}^{i}(y)-d \lambda^{i}(y)\right)\right| d r n(x)=0 .
$$

To see this, for $0<\delta<\Delta$ we write $\int_{0}^{\Delta}=\int_{0}^{\delta}+\int_{\delta}^{\Delta}$. The quantity corresponding to $\int_{0}^{\delta}$ is dominated by $\delta\left(\left\|\mu_{i}^{n}\right\|+\left\|\lambda^{i}\right\|\right) m\left(C_{i}\right)$, and the integrand for $d m(x)$ corresponding to $\int_{\delta}^{\Delta}$ converges to zero boundedly. This proves (3.14) and the proof of the lemma is complete.

LEMMA 3.3. Let $\tau_{n}$ be as in Lemma 3.2. If (1.3), (1.4) hold, then there exists $g_{2}$ : $[0, \infty) \rightarrow[0, \infty)$ such that $\lim _{t \downarrow 0}\left(g_{2}(t) / t\right)=0$, and for every $\Delta>0$, there exists a sequence of Borel measurable functions $\left\{\varphi_{2, n}^{\Delta}\right\}$ which satisfies (2.11), such that for 
$x \in \mathbf{R}^{d}$

$$
P^{x}\left\{\tau_{n} \leqslant \Delta\right\} \geqslant h_{\Delta}(x)-g_{2}(\Delta)-\varphi_{2, n}^{\Delta}(x),
$$

where $h_{\Delta}$ is defined in (2.13).

Proof. With the same notation as in the proof of Lemma 3.2, by (1.4) we have $\left(\nu_{n}^{i}=\nu_{n}^{B(i)}, \varepsilon_{n}^{i}=\varepsilon_{n}^{B(i)}\right)$

$$
\begin{aligned}
P^{x}\left\{\tau_{n} \leqslant \Delta\right\} \geqslant & P^{x}\left\{\tau_{n}^{i} \leqslant \Delta\right\}=\varphi_{x, n}^{i}\left(D_{n}^{i}\right) \\
\geqslant & \int_{D_{n}^{i}}\left(\operatorname{Pot} \nu_{n}^{i}-\operatorname{Pot} \lambda^{i}-\varepsilon_{n}^{i}\right) d \varphi_{x, n}^{i} \\
\geqslant & \int \operatorname{Pot} \varphi_{x, n}^{i} d \nu_{n}^{i}-\int \operatorname{Pot} \varphi_{x, n}^{i} d \lambda^{i}-\varepsilon_{n}^{i} \\
= & \int\left(\operatorname{Pot} \varphi_{x, n}^{i}-\operatorname{Pot} \psi_{x, n}^{i}\right) d \nu_{n}^{i}+\int \operatorname{Pot} \psi_{x, n}^{i} d \nu_{n}^{i} \\
& -\int \operatorname{Pot} \varphi_{x, n}^{i} d \lambda^{i}-\varepsilon_{n}^{i} .
\end{aligned}
$$

By (1.4) Pot $\nu_{n}^{i}$ is finite everywhere, so $\nu_{n}^{i}$ must vanish on a set of capacity zero. Then by (3.3)

$$
\int\left(\operatorname{Pot} \varphi_{x, n}^{i}-\operatorname{Pot} \psi_{x, n}^{i}\right) d \nu_{n}^{i}=\iint_{0}^{\Delta} p(t, x, y) d t d \nu_{n}^{i}(y)
$$

Therefore

$$
P^{x}\left\{\tau_{n} \leqslant \Delta\right\} \geqslant h_{\Delta}(x)-\mathrm{I}_{n}^{i}(x)-\mathrm{II}_{n}^{i}(x)-\mathrm{III}_{n}^{i}(x)-\varepsilon_{n}^{i}
$$

where

$$
\begin{aligned}
\mathrm{I}_{n}^{i}(x) & =\iint_{0}^{\Delta} p(t, x, y) d t\left(d \lambda^{i}(y)-d \nu_{n}^{i}(y)\right) \\
\mathrm{II}_{n}^{i}(x) & =\int \operatorname{Pot} \psi_{x, n}^{i}\left(d \lambda^{i}-d \nu_{n}^{i}\right)
\end{aligned}
$$

and

$$
\begin{aligned}
\operatorname{III}_{n}^{i}(x) & =\int_{\left[\tau_{n}^{i} \leqslant \Delta\right]}\left(\int_{\tau_{n}^{i}}^{\Delta} h\left(B_{s}\right) d s\right) d P^{x} \\
& \leqslant\|h\| \Delta P^{x}\left\{\tau_{n}^{i} \leqslant \Delta\right\} \leqslant\|h\| \Delta\left(h_{\Delta}(x)+g_{1}(\Delta)+\varphi_{1, n}^{\Delta}(x)\right),
\end{aligned}
$$

by Lemma 3.2. Thus

$$
\mathrm{III}_{n}^{i}(x) \leqslant\|h\|^{2} \Delta^{2}+\|h\| \Delta g_{1}(\Delta)+\|h\| \Delta \varphi_{1, n}^{\Delta}(x) .
$$

We set

$$
g_{2}(\Delta)=\|h\|^{2} \Delta^{2}+\|h\| \Delta g_{1}(\Delta)
$$


and

$$
\varphi_{2, n}^{\Delta}(x)=\sum_{i} \chi_{C_{i}}(x)\left[\left(\left|\mathbf{I}_{n}^{i}\right|+\left|\mathrm{II}_{n}^{i}\right|\right) \wedge(1+\|h\| \Delta)\right]+\|h\| \Delta \varphi_{1, n}^{\Delta}(x) .
$$

As in the proof of Lemma 3.2 we have

$$
\int_{C_{i}}\left(\left|\mathrm{I}_{n}^{i}(x)\right|+\left|\mathrm{II}_{n}^{i}(x)\right|\right) d m(x) \rightarrow 0 \text { as } n \rightarrow \infty,
$$

so the lemma is proved.

Getting back to the proof of Theorem 1.2, by Lemma 3.2 we have for any $\Delta>0$, $x \in \mathbf{R}^{d}$

$$
P^{x}\left\{\tau_{n}=0\right\} \leqslant h_{\Delta^{2}}(x)+g_{1}\left(\Delta^{2}\right)+\varphi_{1, n}^{\Delta^{2}}(x) \leqslant\|h\| \Delta^{2}+g_{1}\left(\Delta^{2}\right)+\varphi_{1, n}^{\Delta^{2}}(x),
$$

which implies (2.30) at once, because $P^{x}\left\{A_{n}(\{0\})>\varepsilon\right\}=P^{x}\left\{\tau_{n}=0\right\}$ in the present context. Furthermore, Lemmas 3.2 and 3.3 give us

$$
\left|P^{x}\left\{\tau_{n} \leqslant \Delta\right\}-h_{\Delta}(x)\right| \leqslant g_{1}(\Delta)+g_{2}(\Delta)+\varphi_{1, n}^{\Delta}+\varphi_{2, n}^{\Delta}
$$

and comparing this with (2.14) shows that Condition $\mathrm{A}$ is satisfied by $\left\{P^{x} \otimes A_{n}\right.$ : $\left.x \in \mathbf{R}^{d}, n \geqslant 1\right\}$. This completes the proof of Theorem 1.2.

Remark 3.1. We have shown that both Condition A for the family $\left\{P^{x} \otimes A_{n}\right.$ : $\left.x \in \mathbf{R}^{d}, n \geqslant 1\right\}$ and (2.30) are implied by the single condition: there exists $g$ : $[0, \infty) \rightarrow[0, \infty)$ such that $\lim _{t \downarrow 0}(g(t) / t)=0$ and for each $\Delta>0$, there exists a sequence of Borel measurable functions $\varphi_{n}^{\Delta}: \mathbf{R}^{d} \rightarrow[0, \infty)$ which satisfies (2.11), and for all $x \in \mathbf{R}^{d}, \Delta>0$

$$
\left|P^{x}\left\{\tau_{n} \leqslant \Delta\right\}-h_{\Delta}(x)\right| \leqslant g(\Delta)+\varphi_{n}^{\Delta}(x) .
$$

Lemmas 3.2 and 3.3 show that this condition is satisfied whenever (1.3) and (1.4) hold.

Lemma 3.4. If $\lambda\left(\mathbf{R}^{d}\right)<\infty$, then condition (1.5) implies (1.3) and (1.6) implies (1.4).

Proof. Assume (1.5). Let $\delta_{j} \downarrow 0$. Let $B$ be a bounded ball and let $B_{j}$ be the ball with the same center as $B$ and radius equal to $r+\delta_{j}$, where $r$ is the radius of $B$. We have on $D_{n}$

$$
\operatorname{Pot} \mu_{n}=\operatorname{Pot} \chi_{B_{j}} \mu_{n}+\operatorname{Pot} \chi_{B_{j}^{\prime}} \mu_{n} \geqslant 1+\operatorname{Pot} \lambda-\varepsilon_{n} \text { q.e. }
$$

Since $\mu_{n} \stackrel{n^{\prime}}{\rightarrow} \lambda$, and the distance between $B_{j}^{c}$ and $B$ is $\delta_{j}>0$, we have Pot $\chi_{B_{j}^{c}} \mu_{n} \rightarrow$ Pot $\chi_{B_{j}^{j}} \lambda$ uniformly on $\bar{B}$ as $n \rightarrow \infty$. Therefore there exists a sequence of positive integers $n_{j} \uparrow \infty$ such that for $n_{j} \leqslant n$, on the set $D_{n} \cap \bar{B}$ we have

$$
\operatorname{Pot} \chi_{B_{j}} \mu_{n} \geqslant 1+\operatorname{Pot} \lambda-\operatorname{Pot} \chi_{B_{j}} \lambda-2 \varepsilon_{n} \geqslant 1+\operatorname{Pot} \chi_{B} \lambda-2 \varepsilon_{n} \text {. }
$$

This proves (1.3) if we set $\mu_{n}^{B}=\chi_{B_{1}} \mu_{n}$ for $n_{j} \leqslant n<n_{j+1}$, and $\varepsilon_{n}^{B}=2 \varepsilon_{n}$. For $n<n_{1}$ we simply take $\mu_{n}^{B}=\lambda$ and $\varepsilon_{n}^{B}=1$. 
Now assume (1.6). Proceeding as above, we get $n_{j} \uparrow \infty$ such that for $n_{j} \leqslant n$, on the set $D_{n} \cap \bar{B}$ we have

$$
\begin{aligned}
\operatorname{Pot} \chi_{B_{j}} \nu_{n} & \leqslant 1+\operatorname{Pot} \lambda-\operatorname{Pot} \chi_{B_{j}^{c}} \lambda+2 \varepsilon_{n} \\
& =1+\operatorname{Pot} \chi_{B} \lambda+\operatorname{Pot} \chi_{B_{j}-B} \lambda+2 \varepsilon_{n} .
\end{aligned}
$$

Since $\lambda$ has a bounded density, Pot $\chi_{B_{i}-B} \lambda \rightarrow 0$ uniformly on $\bar{B}$, therefore if $n_{j} \leqslant n$ we have on $D_{n} \cap \bar{B}$

$$
\text { Pot } \chi_{B} \nu_{n} \leqslant \operatorname{Pot} \chi_{B_{1}} \nu_{n} \leqslant 1+\operatorname{Pot} \chi_{B} \lambda+\varepsilon_{n}^{\prime}
$$

where $\varepsilon_{n}^{\prime} \rightarrow 0$ as $n \rightarrow \infty$. For $n_{j} \leqslant n<n_{j+1}$ we set $\nu_{n}^{B}=\chi_{B} \nu_{n}, \varepsilon_{n}^{B}=\varepsilon_{n}^{\prime}$. If $n<n_{1}$, we simply set $\nu_{n}^{B}=0$. This proves the lemma.

Proof of Theorem 1.3. By Lemma 3.4 the conditions (1.5) and (1.6) imply the conditions (1.3), (1.4) of Theorem 1.2. Therefore if $B$ is any bounded ball, then for any $\varepsilon>0$,

$$
\lim _{n \rightarrow \infty} m\left\{x \in B: \sup _{t \leqslant t_{0}}\left\|\nu_{n, t}^{x}-\nu_{t}^{x}\right\|>\varepsilon\right\}=0 .
$$

Without any loss of generality we may assume that

$$
\text { Pot } \mu_{n} \geqslant 1 \text { q.e. on } D_{n}, n \geqslant n_{0},
$$

because if necessary we can replace $\left\{\mu_{n}\right\}$ by the sequence $\left\{\mu_{n}^{\prime}\right\}\left\{\left(1-\varepsilon_{n}^{1 / 2}\right)^{-1} \mu_{n}\right\}$; clearly $\mu_{n}^{\prime} \stackrel{w}{\rightarrow} \lambda$ and Pot $\mu_{n}^{\prime} \geqslant 1+\operatorname{Pot} \lambda$ on $D_{n}$, provided $\varepsilon_{n} \leqslant 1 / 4$.

Let $\varepsilon>0$ be given, $\varepsilon<1$. Since $\mu_{n} \stackrel{w}{\rightarrow} \lambda$, there exists a bounded ball $B$ such that $\mu_{n}\left(B^{c}\right)<\varepsilon^{2} / 2$ for $n \geqslant 1$. Let $\gamma_{n}=\chi_{B^{c}} \mu_{n}$. Let $k(x)=|x|^{2-d}$ if $|x| \leqslant 1, k(x)=0$ otherwise. Let $c=\int k d m$. Clearly

$$
\operatorname{Pot} \gamma_{n}(x) \leqslant \int k(x-y) \gamma_{n}(d y)+\varepsilon^{2} .
$$

Thus, by Chebyshev's inequality, if we let $J_{n}^{\varepsilon}=\left\{x: \operatorname{Pot} \gamma_{n}(x)<\varepsilon\right\}$, then

$$
m\left(\mathbf{R}^{d}-J_{n}^{\varepsilon}\right) \leqslant m\left\{\operatorname{Pot} \gamma_{n} \geqslant \frac{\varepsilon}{2}\right\} \leqslant 2 \int \operatorname{Pot} \gamma_{n} \frac{d m}{\varepsilon}<\frac{c \varepsilon^{2}}{\varepsilon}=c \varepsilon .
$$

Enlarging $B$ if necessary, and letting $K_{n}^{\varepsilon}=\left\{x \in B^{c}\right.$ : Pot $\left.\mu_{n}(x)<2 \varepsilon\right\}$ we have

$$
m\left(B^{c}-K_{n}^{\varepsilon}\right)<c \varepsilon .
$$

Let $\mu_{t}^{x}$ denote the distribution of $B_{t}$ with respect to $P^{x}$. Since $\operatorname{Pot} \mu_{n}\left(B_{t}\right)$ is a continuous supermartingale and $\operatorname{Pot} \mu\left(B_{\tau_{n}}\right) \geqslant 1$ on $\left\{\tau_{n}<\infty\right\}$ (or by the domination principle) we see that

$$
P^{x}\left[\tau_{n}<\infty\right] \leqslant \operatorname{Pot} \mu_{n}(x) \text { for all } x \in \mathbf{R}^{d} .
$$

Therefore if $x \in K_{n}^{\varepsilon}$, we have

$$
\left\|\nu_{n, t}^{x}-\mu_{t}^{x}\right\|<2 \varepsilon, \quad n \geqslant 1, t \geqslant 0 .
$$


We can also make a corresponding argument for $\nu_{t}^{x}$. Let $\gamma=\chi_{B^{c}} \lambda$, where $\lambda\left(B^{c}\right)<$ $\varepsilon^{2} / 2$. Let $J^{\varepsilon}=\{x$ : Pot $\gamma(x)<\varepsilon\}$. As before, $m\left(\mathbf{R}^{d}-J^{\varepsilon}\right)<c \varepsilon$. Enlarging $B$ if necessary, and letting $K^{\varepsilon}=\left\{x \in B^{c}\right.$ : Pot $\left.\lambda(x)<2 \varepsilon\right\}$, we also have

$$
m\left(B^{c}-K^{\varepsilon}\right)<c \varepsilon .
$$

Because $\tau$ is defined as the randomized stopping time with killing rate $h(x)$ at $x$, where $h$ is the density of $\lambda$, it is easy to see that for any $x \in \mathbf{R}^{d}$

$$
P^{x}[\tau<\infty] \leqslant E^{x}\left[\int_{0}^{\infty} h\left(B_{t}\right) d t\right]=\operatorname{Pot} \lambda(x) .
$$

Thus $P^{x}[\tau<\infty]<\varepsilon$ for $x \in K^{\varepsilon}$, so for $x \in K^{\varepsilon}$ we have

$$
\left\|\nu_{t}^{x}-\mu_{t}^{x}\right\|<2 \varepsilon, \quad n \geqslant 1, t \geqslant 0 .
$$

Combining (3.18), (3.22), (3.24) and taking into account (3.20) and (3.23) completes the proof of the theorem.

The following two lemmas will be useful in proving Theorems 1.4 and 1.5 as corollaries of Theorems 1.2 and 1.3 respectively.

LEMma 3.5. Let $\left\{D_{n}\right\}$ satisfy (1.9)(i). Let $\lambda_{i}(n)$ denote the equilibrium measure of $D_{i}(n)$ and let $\lambda_{n}=\sum_{i=1}^{k_{n}} \lambda_{i}(n)$. If for every bounded ball $B, \chi_{B} \lambda_{n} \stackrel{w^{\prime}}{\rightarrow} \chi_{B} \lambda$ as $n \rightarrow \infty$, then for everyy bounded ball $B=B(x, r)$ there exist sequences $0<\varepsilon_{n} \rightarrow 0$ and $0<\delta_{n} \rightarrow 0$ such that, denoting $B(n)=B\left(x, r+3 \delta_{n}\right)$, we have

$$
\operatorname{Pot} \chi_{B(n)} \lambda_{n}>\operatorname{Pot} \chi_{B} \lambda+1-\varepsilon_{n} \quad q . \text {. en the set } D_{n} \cap \bar{B}, n \geqslant 1 \text {. }
$$

Furthermore, if $\lambda\left(\mathbf{R}^{d}\right)<\infty$ and $\lambda_{n} \stackrel{w}{\rightarrow} \lambda$, then there exist $\varepsilon_{n} \rightarrow 0$ such that (3.25) holds with $B=\mathbf{R}^{d}$.

Proof. For $\delta>0$ let $\psi_{\delta}$ be a continuous function on $\mathbf{R}^{d}$ such that $0 \leqslant \psi_{\delta} \leqslant 1$, $\psi_{\delta}=1$ on $B(0, \delta), \psi_{\delta}=0$ outside $B(0,2 \delta)$. Let

$$
\varphi_{\delta}(x)=|x|^{2-d} \psi_{\delta}(x), \quad \varphi_{\delta}^{\prime}(x)=|x|^{2-d}\left(1-\psi_{\delta}(x)\right) .
$$

For a measure $\nu$ we define

$$
\operatorname{Pot}_{\delta} \nu(x)=\int \varphi_{\delta}(x-y) d \nu(y), \quad \operatorname{Pot}_{\delta}^{\prime} \nu(x)=\int \varphi_{\delta}^{\prime}(x-y) d \nu(y) .
$$

Let $\eta_{j}>0, \eta_{j} \rightarrow 0$. Since Pot $\chi_{B} \lambda$ is continuous if $B$ is a bounded ball, given $j$ we can choose $\theta(j), 0<\theta(j)<j^{-1}$, such that

$$
\operatorname{Pot}_{\theta(j)}^{\prime} \chi_{B} \lambda>\operatorname{Pot} \chi_{B} \lambda-\eta_{j} / 2 \text {. }
$$

Since $\chi_{B} \lambda_{n} \stackrel{n}{\rightarrow} \chi_{B} \lambda$, diam $D_{i}(n) \rightarrow 0$ uniformly in $i$, as $n \rightarrow \infty$, and the family of functions $\left\{\varphi_{\theta(j)}^{\prime}(x-\cdot): x \in \mathbf{R}^{d}\right\}$ is uniformly equicontinuous, there exists $n_{j}$ such that for all $x$ and $n \geqslant n_{j}$,

(3.27) $\operatorname{Pot}_{\theta(j)}^{\prime} \chi_{B} \lambda_{n}>\operatorname{Pot}_{\theta(j)}^{\prime} \chi_{B} \lambda-\frac{\eta_{j}}{2}$ and $\max _{1 \leqslant i \leqslant k_{n}} \operatorname{diam} D_{i}(n) \leqslant \theta(j)$. 
By (3.26) and (3.27) we have for all $x$ and $n \geqslant n_{j}$

$$
\operatorname{Pot}_{\theta(j)}^{\prime} \chi_{B} \lambda_{n}>\operatorname{Pot} \chi_{B} \lambda-\eta_{j}, \quad \max _{1 \leqslant i \leqslant k_{n}} \operatorname{diam} D_{i}(n) \leqslant \theta(j)
$$

If $\tilde{B}$ is any ball, and $D_{i}(n) \subset \tilde{B}$, and $n \geqslant n_{j}$, then on the set $D_{i}(n)$ we have

$$
\operatorname{Pot} \chi_{\tilde{B}} \lambda_{n} \geqslant \operatorname{Pot}_{\theta(j)}^{\prime} \chi_{B} \lambda_{n}+\operatorname{Pot} \lambda_{i}(n) \text {. }
$$

If we write $B(j)=B(x, r+3 \theta(j))$, then for $n \geqslant n_{j}, D_{n} \cap \bar{B} \subset \cup_{i}^{\prime} D_{i}(n) \subset \tilde{B}(j)$, where $\bigcup_{i}^{\prime}$ is the union over those $i$ for which $D_{i}(n) \subset \tilde{B}(j)$. Therefore (3.29) implies that for $n \geqslant n_{j}\left(\right.$ since Pot $\lambda_{i}(n)=1$ on $\left.D_{i}(n)\right)$

$$
\operatorname{Pot} \chi_{\tilde{B}(j)} \lambda_{n} \geqslant \operatorname{Pot}_{\theta(j)}^{\prime} \chi_{B} \lambda_{n}+1 \text { on } D_{n} \cap \bar{B} \text {. }
$$

Combining this with (3.28) we get

$$
\operatorname{Pot} \chi_{\tilde{B}(j)} \lambda_{n}>\operatorname{Pot} \chi_{B} \lambda+1-\eta_{j} \text { on } D_{n} \cap \bar{B} \text { for } n \geqslant n_{j} \text {. }
$$

We can clearly take $n_{j} \uparrow$ and define $\delta_{n}=\theta(j), \varepsilon_{n}=\eta_{j}, B(n)=\tilde{B}(j)$ for $n_{j} \leqslant n<$ $n_{j+1}, j \geqslant 1$. Then for $n \geqslant n_{1}$ we have

$$
\text { Pot } \chi_{B(n)} \lambda_{n}>\operatorname{Pot} \chi_{B} \lambda+1-\varepsilon_{n} \text { on } D_{n} \cap \bar{B} \text {. }
$$

Since Pot $\chi_{B} \lambda$ is bounded, for $1 \leqslant n \leqslant n_{1}$ we can pick $\varepsilon_{n}$ so (3.30) holds. This proves (3.25). If $\lambda\left(\mathbf{R}^{d}\right)<\infty$, then the above arguments work with $B=\mathbf{R}^{d}$, and the lemma is proved.

We now define

$$
\lambda_{n}^{B}=\sum_{i}^{*} \lambda_{i}(n), \quad \text { with the sum on those } i \text { for which } D_{i}(n) \subset B \text {. }
$$

Since $\max _{i} \operatorname{diam} D_{i}(n) \rightarrow 0$ and $\lambda$ has a bounded density, $\chi_{B} \lambda_{n} \stackrel{w^{\prime}}{\rightarrow} \chi_{B} \lambda$ implies

$$
\lambda_{n}^{B} \stackrel{w}{\rightarrow} \chi_{B} \lambda
$$

This gives us the following corollary of the proof of Lemma 3.5.

Corollary 3.1. Let $\left\{D_{n}\right\}$ satisfy (1.9) and suppose for each bounded ball $B$, $\chi_{B} \lambda_{n} \stackrel{w}{\rightarrow} \chi_{B} \lambda$. Then there exist $0<\varepsilon_{n} \rightarrow 0$ such that

$$
\operatorname{Pot} \lambda_{n}^{B}>\operatorname{Pot} \chi_{B} \lambda+1-\varepsilon_{n} \text { on } D_{n}^{B}=\bigcup_{i}^{*} D_{i}(n), n \geqslant 1,
$$

where * inaicates union over those $i$ for which $D_{i}(n) \subset B$.

Proof. Following the proof of the lemma we get for $n \geqslant n_{j}$

$$
\operatorname{Pot}_{\theta(j)}^{\prime} \lambda_{n}^{B}>\operatorname{Pot} \chi_{B} \lambda-\eta_{j}, \quad \max _{i} \operatorname{diam} D_{i}(n) \leqslant \theta(j)
$$

and if $D_{i}(n) \subset B$,

$$
\operatorname{Pot} \lambda_{n}^{B} \geqslant \operatorname{Pot}_{\theta(j)}^{\prime} \lambda_{n}^{B}+\operatorname{Pot} \lambda_{i}(n) \text { on } D_{i}(n)
$$

Therefore for $n \geqslant n_{j}$

$$
\text { Pot } \lambda_{n}^{B} \geqslant \operatorname{Pot} \chi_{B} \lambda+1-\eta_{j} \text { on } D_{n}^{B},
$$

taking $\varepsilon_{n}=\eta_{j}$ for $n_{j} \leqslant n<n_{j+1}$, etc., finishes the proof of the corollary. 
LemMa 3.6. Suppose $\left\{D_{n}\right\}$ satisfies (1.9). Then (1.10) and (1.11) imply (1.3) and (1.4). If $\lambda\left(\mathbf{R}^{d}\right)<\infty$, then (1.12) implies (1.5).

Proof. Since $\lambda$ has a bounded density and $\max _{1 \leqslant i \leqslant k_{n}} \operatorname{diam} D_{i}(n) \rightarrow 0$ as $n \rightarrow \infty$, it is easy to see that (1.10) implies that for every bounded ball $B$

$$
\chi_{B} \lambda_{n} \stackrel{\text { w' }}{\rightarrow} \chi_{B} \lambda \quad \text { as } n \rightarrow \infty
$$

where $\lambda_{n}$ is defined in Lemma 3.5. It is also clear that if $\lambda\left(\mathbf{R}^{d}\right)<\infty$, then (1.12) implies (3.33) with $B=\mathbf{R}^{d}$. If we assume (1.10), then (3.25) holds by Lemma 3.5. It is immediate that $\chi_{B_{n}} \lambda_{n} \stackrel{w}{\rightarrow} \chi_{B} \lambda$, so (1.3) holds with $\mu_{n}^{B}=\chi_{B_{n}} \lambda_{n}$. If $\lambda\left(\mathbf{R}^{d}\right)<\infty$ and (1.12) is assumed, then again by Lemma 3.5 we have

$$
\operatorname{Pot} \lambda_{n}>\operatorname{Pot} \lambda+1-\varepsilon_{n} \text { q.e. on } D_{n} \cap \bar{B}
$$

so (1.5) holds with $\mu_{n}=\lambda_{n}$. It thus suffices to check that (1.10) and (1.11) imply (1.4). Let $\psi_{\delta}, \varphi_{\delta}, \psi_{\delta}^{\prime}, \varphi_{\delta}^{\prime}$ be defined as in the proof of Lemma 3.5, and for a measure $\nu$ let

$$
\langle\nu, \nu\rangle_{\delta}=\iint \varphi_{\delta}(x-y) d \nu(x) d \nu(y), \quad\langle\nu, \nu\rangle_{\delta}^{\prime}=\iint \varphi_{\delta}^{\prime}(x-y) d \nu(x) d \nu(y)
$$

By assumption (1.9) we have

$$
C \text { Pot } \mu_{i}(n)(x)=C \alpha_{i}(n) \frac{1}{\left|x-x_{i}(n)\right|^{d-2}} \geqslant 1, \quad x \in B\left(x_{i}(n), \rho_{n}\right),
$$

so by the domination principle

$$
\operatorname{Pot} \lambda_{i}(n) \leqslant C \operatorname{Pot} \mu_{i}(n) \text { on } \mathbf{R}^{d} .
$$

This implies

$$
\left\langle\lambda_{i}(n), \lambda_{j}(n)\right\rangle \leqslant C^{2}\left\langle\mu_{i}(n), \mu_{j}(n)\right\rangle .
$$

Let $B$ be a bounded ball and $\delta>0$. An asterisk on $\sum$ will indicate summation over those $i, j$ for which $D_{i}(n)$ and $D_{j}(n)$ are contained in $B$. Then

$$
\sum_{i \neq j}^{*}\left\langle\mu_{i}(n), \mu_{j}(n)\right\rangle=\sum_{i \neq j}^{*}\left\langle\mu_{i}(n), \mu_{j}(n)\right\rangle_{\delta}+\sum_{i \neq j}^{*}\left\langle\mu_{i}(n), \mu_{j}(n)\right\rangle_{\delta}^{\prime} .
$$

Assume $n$ is large enough that $2 \rho_{n}<\delta$. Since for all $n$ and $i,\left\langle\mu_{i}(n), \mu_{i}(n)\right\rangle_{\delta}^{\prime}=0$, we then have

$$
\sum_{i \neq j}^{*}\left\langle\mu_{i}(n), \mu_{j}(n)\right\rangle=\sum_{i \neq j}^{*}\left\langle\mu_{i}(n), \mu_{j}(n)\right\rangle_{\delta}+\left\langle\mu_{n}^{B}, \mu_{n}^{B}\right\rangle_{\delta}^{\prime}
$$

where $\mu_{n}^{B}=\sum_{i}^{*} \mu_{i}(n)$, sum on those $i$ for which $D_{i}(n) \subset B$. Since $\chi_{B} \mu_{n} \stackrel{w}{\rightarrow} \chi_{B} \lambda, \lambda$ has a bounded density, and $\max _{i} \operatorname{diam} D_{i}(n) \rightarrow 0$ as $n \rightarrow \infty$, it is easily seen that

$$
\mu_{n}^{B} \stackrel{w}{\rightarrow} \chi_{B} \lambda
$$


Consequently, $\left\langle\mu_{n}^{B}, \mu_{n}^{B}\right\rangle_{\delta}^{\prime} \rightarrow\left\langle\chi_{B} \lambda, \chi_{B} \lambda\right\rangle_{\delta}^{\prime}$. Also, $\lim _{\delta \rightarrow 0}\left\langle\chi_{B} \lambda, \chi_{B} \lambda\right\rangle_{\delta}^{\prime}=$ $\left\langle\chi_{B} \lambda, \chi_{B} \lambda\right\rangle$. Applying these facts to (3.35) we conclude that (1.11) holds iff for every $\varepsilon>0$, there is a $\delta(\varepsilon)$ such that for $\delta \leqslant \delta(\varepsilon)$

$$
\limsup _{n} \sum_{i \neq j}^{*}\left\langle\mu_{i}(n), \mu_{j}(n)\right\rangle_{\delta}<\varepsilon .
$$

Suppose (3.37) holds for $\varepsilon>0$ and $\delta>0$. We wish to show that it also holds in an essential manner if $\mu_{i}(n)$ and $\mu_{j}(n)$ are replaced by $\left.\lambda_{i} n\right)$ and $\lambda_{j}(n)$, respectively. If $\left\langle\lambda_{i}(n), \lambda_{j}(n)\right\rangle_{\delta / 3} \neq 0$, then there are points in $D_{i}(n)$ within $\delta / 3$ of $D_{j}(n)$, so $\left|x_{i}(n)-x_{j}(n)\right|<\delta / 3+2 \rho_{n}<\delta$ if $\rho_{n} \leqslant \delta / 3$, and then $\left\langle\mu_{i}(n), \mu_{j}(n)\right\rangle_{\delta}=$ $\left\langle\mu_{i}(n), \mu_{j}(n)\right\rangle$. Therefore

$$
\begin{aligned}
\sum_{i \neq j}^{*}\left\langle\lambda_{i}(n), \lambda_{j}(n)\right\rangle_{\delta / 3} \leqslant & \sum_{\substack{i \neq j \\
\left|x_{i}(n)-x_{j}(n)\right|<\delta}}^{*}\left\langle\lambda_{i}(n), \lambda_{j}(n)\right\rangle \\
\leqslant & \sum_{\substack{i \neq j \\
\left|x_{i}(n)-x_{j}(n)\right|<\delta}}^{*} C^{2}\left\langle\mu_{i}(n), \mu_{j}(n)\right\rangle \\
& \leqslant C^{2} \sum_{i \neq j}^{*}\left\langle\mu_{i}(n), \mu_{j}(n)\right\rangle_{\delta},
\end{aligned}
$$

where (3.34) is used at the second step. Thus (3.37) implies

$$
\lim _{\delta \rightarrow 0} \limsup _{n} \sum_{i \neq j}^{*}\left\langle\lambda_{i}(n), \lambda_{j}(n)\right\rangle_{\delta}=0 .
$$

Let $\lambda_{n}^{B}=\sum_{i}^{*} \lambda_{i}(n)$, sum on those $i$ for which $D_{i}(n) \subset B$. In view of (3.33), for the same reason that applied to (3.36) we get

$$
\lambda_{n}^{B} \stackrel{w}{\rightarrow} \chi_{B} \lambda .
$$

Therefore $\left\langle\lambda_{n}^{B}, \lambda_{n}^{B}\right\rangle_{\delta}^{\prime} \rightarrow\left\langle\chi_{B} \lambda, \chi_{B} \lambda\right\rangle_{\delta}^{\prime}$. Also

$$
\begin{aligned}
\left\langle\lambda_{n}^{B}, \lambda_{n}^{B}\right\rangle & =\left\langle\lambda_{n}^{B}, \lambda_{n}^{B}\right\rangle_{\delta}+\left\langle\lambda_{n}^{B}, \lambda_{n}^{B}\right\rangle_{\delta}^{\prime} \\
& =\sum_{i \neq j}^{*}\left\langle\lambda_{i}(n), \lambda_{j}(n)\right\rangle_{\delta}+\sum_{i}^{*}\left\langle\lambda_{i}(n), \lambda_{i}(n)\right\rangle_{\delta}+\left\langle\lambda_{n}^{B}, \lambda_{n}^{B}\right\rangle_{\delta}^{\prime} .
\end{aligned}
$$

For $n$ sufficiently large

$$
\left\langle\lambda_{i}(n), \lambda_{i}(n)\right\rangle_{\delta}=\int_{D_{i}(n)} \operatorname{Pot} \lambda_{i}(n)(x) d \lambda_{i}(n)(x)=\lambda_{i}(n)\left(\mathbf{R}^{d}\right) .
$$

Therefore, if we let $n \rightarrow \infty$, and then $\delta \rightarrow 0$ in (3.40) and use (3.38) we get

$$
\lim _{n \rightarrow \infty}\left\langle\lambda_{n}^{B}, \lambda_{n}^{B}\right\rangle=\left\langle\chi_{B} \lambda, \chi_{B} \lambda\right\rangle+\lambda(B) .
$$

By (3.39), (3.41) and Corollary 3.1 we can pick $\varepsilon_{n} \rightarrow 0$ such that

$$
\begin{gathered}
\left|\left\langle\lambda_{n}^{B}, \lambda_{n}^{B}\right\rangle-\left\langle\chi_{B} \lambda, \chi_{B} \lambda\right\rangle-\lambda(B)\right|<\varepsilon_{n}^{2}, \\
\left|\left\langle\chi_{B} \lambda, \chi_{B} \lambda\right\rangle-\left\langle\chi_{B} \lambda, \lambda_{n}^{B}\right\rangle\right|<\varepsilon_{n}^{2}, \\
\left|\lambda(B)-\lambda_{n}^{B}(B)\right|<\varepsilon_{n}^{2},
\end{gathered}
$$


and

$$
\operatorname{Pot} \lambda_{n}^{B}>\operatorname{Pot} \chi_{B} \lambda+1-\varepsilon_{n}^{2} \text { on } D_{n}^{B}=\bigcup_{i}^{*} D_{i}(n),
$$

where $*$ denotes union over those $i$ for which $D_{i}(n) \subset B$. Let

$$
\Gamma_{n}=\left\{x: \operatorname{Pot} \lambda_{n}^{B} \geqslant \operatorname{Pot} \chi_{B} \lambda+1+\varepsilon_{n}\right\} .
$$

Then, noting that $D_{n}^{B}$ supports $\lambda_{n}^{B}$, we have by (3.43)-(3.45),

$$
\begin{aligned}
\left\langle\lambda_{n}^{B}, \lambda_{n}^{B}\right\rangle & \geqslant \int_{\Gamma_{n} \cap D_{n}^{B}}\left(\operatorname{Pot} \chi_{B} \lambda+1+\varepsilon_{n}\right) d \lambda_{n}^{B}+\int_{\Gamma_{n}^{C} \cap D_{n}^{B}}\left(\operatorname{Pot} \chi_{B} \lambda+1-\varepsilon_{n}^{2}\right) d \lambda_{n}^{B} \\
& =\left\langle\chi_{B} \lambda, \lambda_{n}^{B}\right\rangle+\lambda_{n}^{B}(B)+\varepsilon_{n} \lambda_{n}^{B}\left(\Gamma_{n}\right)-\varepsilon_{n}^{2} \lambda_{n}^{B}\left(\Gamma_{n}^{C} \cap D_{n}^{B}\right) \\
& \geqslant\left\langle\chi_{B} \lambda, \chi_{B} \lambda\right\rangle-\varepsilon_{n}^{2}+\lambda(B)-\varepsilon_{n}^{2}+\varepsilon_{n} \lambda_{n}^{B}\left(\Gamma_{n}\right)-\varepsilon_{n}^{2} \lambda_{n}(B) .
\end{aligned}
$$

Therefore by (3.42) we have

$$
\varepsilon_{n} \lambda_{n}^{B}\left(\Gamma_{n}\right) \leqslant \varepsilon_{n}^{2}\left(3+\lambda_{n}(B)\right) .
$$

Let $\nu_{n}=\chi_{\Gamma_{n}^{\prime}} \lambda_{n}^{B}$. Then

$$
\text { Pot } \nu_{n} \leqslant \operatorname{Pot} \lambda_{n}^{B}<\operatorname{Pot} \chi_{B} \lambda+1+\varepsilon_{n} \text { on } \Gamma_{n}^{c}
$$

but $\nu_{n}$ has support in $\Gamma_{n}^{c}$, so (3.47) holds everywhere by the domination principle. Since $\lambda_{n}^{B}$ has support in $D_{n} \cap \bar{B}, \nu_{n}$ has support in $D_{n} \cap \bar{B}$. Also (3.46) and (3.39) imply that $\nu_{n} \stackrel{n^{\prime}}{\rightarrow} \chi_{B} \lambda$. This shows that (1.4) holds with $\nu_{n}^{B}=\nu_{n}$ and the lemma is proved.

Theorems 1.4 and 1.5 now follow immediately from Theorems 1.2 and 1.3, respectively, via Lemma 3.6.

\section{REFERENCES}

1. J. R. Baxter and R. V. Chacon, Compactness of stopping times, Z. Wahrsch. Verw. Gebiete 40 (1977), $169-181$.

2. $1055-1065$.

. Enlargement of o-algebras and compactness of time changes, Canad. J. Math. 29 (1977),

3. J. L. Doob, Classical potential theory and its probabilistic counterpart, Springer-Verlag, New York, 1984.

4. G. A. Edgar, A. Millet and L. Sucheston, On compactness and optimality of stopping times, Proc. Conf. on Martingale Theory in Harmonic Analysis and Banach Spaces, Lecture Notes in Math., vol. 939. Springer-Verlag, Berlin, 1982, pp. 36-61.

5. P. A. Meyer, Convergence faible et compacité des temps d'arrět, d'après Baxter et Chacon, Séminaire de Probabilités XII, Lecture Notes in Math., vol. 649, Springer-Verlag, New York, 1978, pp. 411-423.

6. (3. C. Papanicolaou and S. R. S. Varadhan, Diffusions in regions with many small holes, Stochastic Differential Systems-Filtering and Control (B. Grigelionis, ed.), Lecture Notes in Control and Information Sciences, vol. 25, Springer-Verlag, New York, 1980, pp. 190-206.

7. J. Rauch and M. Taylor, Potential and scattering theory on wildly perturbed domains, J. Funct. Anal. 18 (1975), 27-59.

Department of Mathematics, University of Minnesota, Minneapol.is, Minnesota 55455 (Current address of J. R. Baxter and N. C. Jain)

Department of Mathematics, University of British Columbia, Vancouver, British Columbia, CANADA (Current address of R. V. Chacon) 